\section{Rotation Method for Direction Finding via GPS Carrier Phases}

\author{
H. M. PENG \\ F. R. CHANG \\ L. S. WANG \\ National Taiwan University
}

\begin{abstract}
A baseline rotation method is proposed for determining the direction of the baseline vector via Global Positioning System (GPS) carrier phase measurements. The space difference technique is adopted to resolve GPS carrier phase cycle ambiguities. Possible applications include the determination of the aiming directions of artillery rockets and the line of sights of tracking radars, etc. For such armaments, the direction findings are important and the rotating mechanisms are well equipped already. A general baseline vector which can be rotated on a two-degree-of-freedom platform is considered first. The relationship among the baseline vector and the two rotation axes is not known. A sequence of rotations is used to change the configuration of the system to find the direction of the baseline. Under different circumstances such as the cases that some orthogonal conditions among the unknown vectors are given, simplified algorithms are devised. To verify our method, software simulation and hardware experiments have been conducted. The simulation outcomes are used to determine the experimental parameters, such as the length of the baseline, the rotation angles etc. The results of repeated hardware experiments show that the sample standard deviation for the azimuth angle and the elevation angle of the $1.35 \mathrm{~m}$ baseline vector are $0.91 \mathrm{deg}$ and $1.23 \mathrm{deg}$, respectively. The GPS receivers employed are Motorola ONCOREs. The errors of the estimated direction angles induced by the inaccuracy of rotation angles, which are unavoidable due to the imperfectness of the mechanical structure, are analyzed as well. Numerical examples for the error analysis are included.
\end{abstract}

Manuscript received October 13, 1997; revised December 24, 1998 and September 7, 1999; released for publication October 22, 1999.

Refereeing of this contribution was handled by X. R. Li.

IEEE Log No. T-AES/36/1/01406.

This research was supported by the National Science Council of the R.O.C. under Grant NSC 87-2612-E002-002.

Authors' addresses: H. M. Peng and F. R. Chang, Dept. of Electrical Engineering, National Taiwan University, No. 1, Sec. 4, Roosevelt Rd., Taipei, Taiwan, R.O.C.; L. S. Wang, Institute of Applied Mechanics, National Taiwan University, Taipei, Taiwan, R.O.C., E-mail: (frchang@ac.ee.ntu.edu.tw).

$\overline{0018-9251 / 00 / \$ 10.00 ~(c) ~} 2000$ IEEE

\section{INTRODUCTION}

The problems of direction finding frequently arise, such as in the navigation and control of vehicles, in solving triangulation problems in surveying, in aiming of artillery rockets, and in target acquisition of tracking radars. The compass and the gyroscope are the most popular sensors to obtain the direction information. Some other ground-based navigation systems like the VHF Omni Range (VOR) and the radio beacons are also used in direction findings. All these sensors have some defects, however. The compass points to the magnetic north of the Earth instead of the true north. The reading of a compass is sensitive to the nearby magnetic field. The drift problem, i.e., the accumulation of errors, associated with the gyroscope makes it necessary to calibrate periodically. On the other hand, the VOR and radio beacons have range limitations. The advent of Global Positioning System (GPS) provides another route to solve the problems of direction finding. GPS does not have any of the problems that plague the sensors mentioned above.

Considering the accuracy of direction finding, the GPS carrier phase observables must be used. Based on the idea of interferometry, several algorithms for direction finding or attitude determination have been developed in [2-8]. The main difficulty of using carrier phase measurements is to find the initial ambiguities. This problem has been investigated by many researchers. Various methodologies have been proposed, such as the ambiguity search technique [3-4], the ambiguity function method [5], the time-difference method [6], and the space-difference method [6-8]. The first two methods need the double differences of carrier phase observables. The integer ambiguity problem is solved by search algorithms either on the space of possible ambiguous values or on the space of possible coordinates. The performance of these methods depends highly on the quality of the GPS receivers. For low-cost single-frequency GPS receivers, the convergent time to obtain the true direction may be very long. Time-difference algorithms use the triple differences of observables, and are time consuming in determining the initial state. The space-difference methods are an extension of the ambiguity methods that use double differences; in particular, they gain additional observability to the initial ambiguities by induced motion in the baseline. As for space-difference methods, the antenna-swap approach was used early in the surveying field and other schemes were discussed in [6].

The rotation method proposed here belongs to space-difference approaches. The baseline vector is rotated about two axes in given angles, and carrier phase observables are measured before and after every rotation. The differences between epochs, during which the configuration has been changed, 
are computed on the double differences of phase observables to obtain ambiguity-free measurements. Through the rotations of the structure, the rotation axes and the direction vector can be then solved. The double-difference integer ambiguities can be found immediately as well. Comparing with our baseline rotation method, the antenna-swap approach can be considered a special case as the rotation angles are $180 \mathrm{deg}$. The scheme discussed in [6] uses an inner product relationship between adjacent phase measurements to solve the problem. However, the rotation method proposed here is more suitable if some rotational mechanism already existed, such as the launchers of the artillery rockets and the antennas of tracking radars, etc.

To verify our algorithms, simulations and experiments have been conducted. In simulations, the white noises are added to phase measurements to evaluate the effects caused by the changes of the baseline length, the rotation angles, the initial baseline azimuth, or the elevation angles. The simulation results show that the baseline length, the rotation angles, and the initial baseline elevation angles have significant effects on the errors for direction finding, but the initial baseline azimuth angle does not. Based on the simulation outcomes, an appropriate experimental configuration was devised. A two-degree-of-freedom platform with a $1.35 \mathrm{~m}$ aluminum bar representing the baseline vector was constructed for hardware experiments. By processing the carrier phase observables from the two antennas attached to both ends of the bar, the proposed algorithms computed the direction vector along the bar. In our experiment, three rotations are enough to determine the unknown direction vector by using the baseline rotation method. In our proposed method, the azimuth and elevation angles rotated must be known for the integer ambiguity resolution. The azimuth angle of the baseline vector relative to the local north direction and the elevation angle relative to the local horizontal plane are the results of our algorithm. They are unknown variables in advance. Two Motorola ONCORE 8-channel GPS receivers were used to collect data in the experiments. After 12 consecutive trials, the sample standard deviation for the azimuth angle and the elevation angle of the direction vector are about $0.91 \mathrm{deg}$ and $1.23 \mathrm{deg}$, respectively. With longer length of the baseline vector, the errors can be reduced.

This paper is organized as follows. In Section II, the GPS carrier phase double-difference model is briefly described. The rotation method for a general two-degree-of-freedom baseline direction finding is discussed in Section III. Using special relationships among the rotation axes and the baseline vector, the algorithm proposed in Section III can be simplified. Two special cases are discussed in Section IV. The simulation results and the experimental data are given

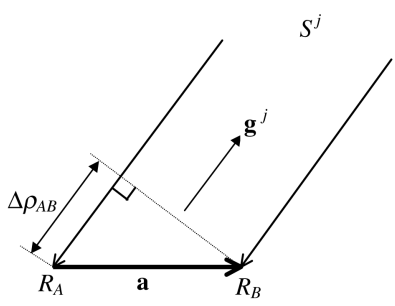

Fig. 1. Direction finding via GPS carrier phases.

in Section V. In Section VI, an error analysis has been performed to find out the effects caused by the errors in the rotation angles. It is shown that with \pm 1 deg errors in rotation angles, the errors for the azimuth angle and the elevation angle of the direction vector are about $0.5 \mathrm{deg}$ and $0.8596 \mathrm{deg}$, respectively. Conclusions are given in Section VII.

\section{GPS CARRIER PHASE DOUBLE-DIFFERENCE MODEL}

The GPS carrier phase observable counts the beat phase, i.e., the difference between the L-band carrier wave from a satellite and the reference signal generated by the receiver. It can be modeled as

$$
\phi_{A}^{j}=\rho_{A}^{j}+c\left(\delta t^{j}-\delta T_{A}\right)+\lambda N_{A}^{j}-d_{\text {ion }}^{j}+d_{\text {trop }}^{j}+\varepsilon_{A}^{j}
$$

where $\phi_{A}^{j}$ is the carrier phase measurements of the receiver $A$ from the $j$ th GPS satellite; $\rho_{A}^{j}$ is the true distance between the receiver $A$ and the $j$ th GPS satellite; $c$ is the speed of light; $\delta t^{j}$ and $\delta T_{A}$ are the clock biases of the $j$ th satellite and the receiver $A$, respectively; $\lambda$ is the GPS carrier wavelength; $N_{A}^{j}$ denotes the initial phase integer ambiguity; and $d_{\text {ion }}^{j}, d_{\text {trop }}^{j}$, and $\varepsilon_{A}^{j}$ are the ionospheric delay, the tropospheric delay, and the unmodeled errors, respectively. The unit of the phase observable $\phi_{A}^{j}$ in the equation is meters.

To eliminate the biases and the errors from satellites and receivers, it is customary to perform double differences between two receivers and two satellites. Denoting the two receivers as $A$ and $B$ and the satellites as $j$ and $k$, the double-difference equation is

$$
\nabla \Delta \phi_{A B}^{j k}=\nabla \Delta \rho_{A B}^{j k}+\lambda \nabla \Delta N_{A B}^{j k}+\nabla \Delta \varepsilon_{A B}^{j k}
$$

where the convention $\nabla \Delta *_{A B}^{j k}=\left(*_{B}^{k}-*_{A}^{k}\right)-\left(*_{B}^{j}-*_{A}^{j}\right)$ is used, and the asterisk may be replaced by $\phi, \rho, N$, and $\varepsilon$. The satellite clock bias, the receiver clock bias, the ionospheric delay, and the tropospheric delay are then eliminated in the double-difference model.

The concept of interferometry is described in the following. In Fig. $1, R_{A}$ and $R_{B}$ denote two GPS antennas which are mounted on the two ends of the baseline vector a, respectively. They receive signals from the same GPS satellite denoted as $S^{j}$ with 


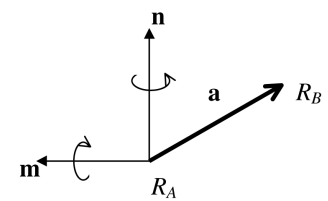

Fig. 2. Configuration of two-axis platform.

the same unit directional vector $\mathbf{g}^{j}$. The difference between the distances from the two antennas to the satellite $S^{j}$ is then

$$
\Delta \rho_{A B}=\left(\mathbf{g}^{j}\right)^{\mathrm{T}} \mathbf{a}
$$

where the superscript $\mathrm{T}$ denotes the transpose.

With $n$ satellites $(n \geq 4)$ being observed at the same time, the matrix form of the equations can be written from (2) and (3) as

where

$$
\Phi=\mathbf{G}^{\mathrm{T}} \mathbf{a}+\lambda \mathbf{N}+\mathbf{E}
$$

$$
\begin{aligned}
\mathbf{G} & =\left[\begin{array}{llll}
\mathbf{g}^{2}-\mathbf{g}^{1} & \mathbf{g}^{3}-\mathbf{g}^{1} & \cdots & \mathbf{g}^{n}-\mathbf{g}^{1}
\end{array}\right] \\
\Phi & =\left[\begin{array}{llll}
\nabla \Delta \phi_{A B}^{12} & \nabla \Delta \phi_{A B}^{13} & \cdots & \nabla \Delta \phi_{A B}^{1 n}
\end{array}\right]^{\mathrm{T}} \\
\mathbf{N} & =\left[\begin{array}{llll}
\nabla \Delta N_{A B}^{12} & \nabla \Delta N_{A B}^{13} & \cdots & \nabla \Delta N_{A B}^{1 n}
\end{array}\right]^{\mathrm{T}} \\
\mathbf{E} & =\left[\begin{array}{llll}
\nabla \Delta \varepsilon_{A B}^{12} & \nabla \Delta \varepsilon_{A B}^{13} & \cdots & \nabla \Delta \varepsilon_{A B}^{1 n}
\end{array}\right]^{\mathrm{T}} .
\end{aligned}
$$

The main difficulty in using this model is on the determination of the initial integer ambiguities N. A method resolving this problem is presented next.

\section{BASELINE ROTATION METHOD}

In the following discussion, the unmodeled error $\varepsilon_{A}^{j}$ in (1) is assumed as the sum of two sources $b_{A}^{j}$ and $w_{A}^{j}$, where $b_{A}^{j}$ is a deterministic bias term, and $w_{A}^{j}$ is a white noise that has the normal density function $\mathcal{N}\left(0, \sigma^{2}\right)$.

Consider the configuration shown in Fig. 2. The unknown baseline vector a is formed by two GPS receivers $R_{A}$ and $R_{B}$ and can be rotated about the axes $\mathbf{n}$ and $\mathbf{m}$, respectively. The rotation axes $\mathbf{m}$ and $\mathbf{n}$ are unit vectors and the length of the vector $\mathbf{a}$ is assumed to be a known value $l(=|\mathbf{a}|)$.

The detail of the baseline rotation method is described in the following. Considering the general case, in which the baseline vector $\mathbf{a}$, the rotation axis $\mathbf{n}$ and the axis $\mathbf{m}$ are unknown and no relationship exists between them. It is shown that two successive rotations about the axis $\mathbf{n}$ by the two given angles $\theta_{1}$ and $\theta_{2}$, and followed by two successive rotations about the axis $\mathbf{m}$ by the two given angles $\varphi_{1}$ and $\varphi_{2}$ are adequate for determining the baseline vector $\mathbf{a}$ as well as $\mathbf{m}$ and $\mathbf{n}$. The phase observables are received before and after every rotation. In addition, the direction cosine matrix $\mathbf{G}$ in (4) is assumed to be the same during the rotational processes.
Let

$$
\begin{aligned}
& \mathbf{a}_{1}=\mathbf{a} \\
& \mathbf{a}_{i}=\mathbf{R}\left(\mathbf{n}, \theta_{i-1}\right) \mathbf{a}, \quad i=2,3 \\
& \mathbf{a}_{i}=\mathbf{R}\left(\mathbf{m}, \varphi_{i-3}\right) \mathbf{a}, \quad i=4,5
\end{aligned}
$$

where $\mathbf{R}(\mathbf{n}, \theta)$ is the rotation matrix about the rotation axis $\mathbf{n}$ by the rotation angle $\theta$. The Euler's formula for rotation matrix $\mathbf{R}$ given in [10] is used later

$$
\mathbf{R}(\mathbf{n}, \theta)=\mathbf{I}-\sin \theta[|\mathbf{n}|]+(1-\cos \theta)[|\mathbf{n}|]^{2}
$$

where

$$
[|\mathbf{n}|]=\left[\begin{array}{ccc}
0 & n_{3} & -n_{2} \\
-n_{3} & 0 & n_{1} \\
n_{2} & -n_{1} & 0
\end{array}\right] \quad \text { for } \quad \mathbf{n}=\left[\begin{array}{l}
n_{1} \\
n_{2} \\
n_{3}
\end{array}\right] .
$$

According to the carrier phase double-difference model (4), the five carrier phase observables for different baseline vectors $a_{i}, i=1, \ldots, 5$ can be modeled as

$$
\begin{aligned}
\Phi_{1} & =\mathbf{G}^{\mathrm{T}} \mathbf{a}_{1}+\lambda \mathbf{N}+\mathbf{B}+\mathbf{W}_{1}=\mathbf{G}^{\mathrm{T}} \mathbf{a}+\lambda \mathbf{N}+\mathbf{B}+\mathbf{W}_{1} \\
\Phi_{i} & =\mathbf{G}^{\mathrm{T}} \mathbf{a}_{i}+\lambda \mathbf{N}+\mathbf{B}+\mathbf{W}_{i} \\
& =\mathbf{G}^{\mathrm{T}} \mathbf{R}\left(\mathbf{n}, \theta_{i-1}\right) \mathbf{a}+\lambda \mathbf{N}+\mathbf{B}+\mathbf{W}_{i}, \quad i=2,3 \\
\Phi_{i} & =\mathbf{G}^{\mathrm{T}} \mathbf{a}_{i}+\lambda \mathbf{N}+\mathbf{B}+\mathbf{W}_{i} \\
& =\mathbf{G}^{\mathrm{T}} \mathbf{R}\left(\mathbf{m}, \varphi_{i-3}\right) \mathbf{a}+\lambda \mathbf{N}+\mathbf{B}+\mathbf{W}_{i}, \quad i=4,5
\end{aligned}
$$

where

$$
\begin{aligned}
B & =\left[\begin{array}{lll}
\nabla \Delta b_{A B}^{12} & \cdots & \nabla \Delta b_{A B}^{1 n}
\end{array}\right]^{\mathrm{T}} \quad \text { and } \\
W_{i} & =\left[\begin{array}{lll}
\left(\nabla \Delta w_{A B}^{12}\right)_{i} & \cdots & \left(\nabla \Delta w_{A B}^{1 n}\right)_{i}
\end{array}\right]^{\mathrm{T}} .
\end{aligned}
$$

Let $\mathbf{Q}$ denote the covariance matrix of the double-difference carrier phase observables. Its inverse $\mathbf{Q}^{-1}$ can be used as the weighting for different observations. The standard weighted least square method then can be applied to compute the intermediate vector $\mathbf{a}_{i m}$ as

$$
\mathbf{a}_{i m}=\left(\mathbf{G} \mathbf{Q}^{-1} \mathbf{G}^{\mathrm{T}}\right)^{-1} \mathbf{G Q}^{-1} \Phi_{i}, \quad i=1, \ldots, 5
$$

where $\Phi_{i}$ are as in (7). In order to eliminate the integer ambiguities, further differences are performed as

$$
\begin{aligned}
& \mathbf{a}_{1 i m}= \mathbf{a}_{i m}-\mathbf{a}_{1 m}=\left(\mathbf{G} \mathbf{Q}^{-1} \mathbf{G}^{\mathrm{T}}\right)^{-1} \mathbf{G} \mathbf{Q}^{-1}\left(\Phi_{i}-\Phi_{1}\right) \\
&=\left(\mathbf{R}\left(\mathbf{n}, \theta_{i-1}\right)-\mathbf{I}\right) \mathbf{a}+\left(\mathbf{G} \mathbf{Q}^{-1} \mathbf{G}^{\mathrm{T}}\right)^{-1} \mathbf{G} \mathbf{Q}^{-1}\left(\mathbf{W}_{i}-\mathbf{W}_{1}\right), \\
& i=2,3 \\
& \mathbf{a}_{1 i m}=\mathbf{a}_{i m}-\mathbf{a}_{1 m}=\left(\mathbf{G} \mathbf{Q}^{-1} \mathbf{G}^{\mathrm{T}}\right)^{-1} \mathbf{G} \mathbf{Q}^{-1}\left(\Phi_{i}-\Phi_{1}\right) \\
&=\left(\mathbf{R}\left(\mathbf{m}, \varphi_{i-3}\right)-\mathbf{I}\right) \mathbf{a}+\left(\mathbf{G} \mathbf{Q}^{-1} \mathbf{G}^{\mathrm{T}}\right)^{-1} \mathbf{G} \mathbf{Q}^{-1}\left(\mathbf{W}_{i}-\mathbf{W}_{1}\right), \\
& i=4,5 .
\end{aligned}
$$


Taking the sample averages on the above equations, the white noises can be mostly eliminated, so that

$$
\begin{aligned}
& \overline{\mathbf{a}}_{1 i m}=\left(\mathbf{R}\left(\mathbf{n}, \theta_{i-1}\right)-\mathbf{I}\right) \mathbf{a}, \quad i=2,3 \\
& \overline{\mathbf{a}}_{1 i m}=\left(\mathbf{R}\left(\mathbf{m}, \varphi_{i-3}\right)-\mathbf{I}\right) \mathbf{a}, \quad i=4,5
\end{aligned}
$$

where the symbol $\overline{\mathbf{a}}$ means the sample average of the vector a. Since

$$
\begin{aligned}
\overline{\mathbf{a}}_{1 i m}^{\mathrm{T}} \mathbf{n} & =\mathbf{a}^{\mathrm{T}}\left(\mathbf{R}\left(\mathbf{n}, \theta_{i-1}\right)-\mathbf{I}\right)^{\mathrm{T}} \mathbf{n} \\
& =\mathbf{a}^{\mathrm{T}}\left(\mathbf{R}\left(\mathbf{n},-\theta_{i-1}\right)-\mathbf{I}\right) \mathbf{n}=0, \quad i=2,3 \\
\overline{\mathbf{a}}_{1 i m}^{\mathrm{T}} \mathbf{m} & =\mathbf{a}^{\mathrm{T}}\left(\mathbf{R}\left(\mathbf{m}, \varphi_{i-3}\right)-\mathbf{I}\right)^{\mathrm{T}} \mathbf{n} \\
& =\mathbf{a}^{\mathrm{T}}\left(\mathbf{R}\left(\mathbf{m},-\varphi_{i-3}\right)-\mathbf{I}\right) \mathbf{m}=0, \quad i=4,5
\end{aligned}
$$

it can be easily seen that $\overline{\mathbf{a}}_{1 i m}, i=2,3$ are orthogonal to the rotation axis $\mathbf{n}$, and $\overline{\mathbf{a}}_{1 i m}, i=4,5$ are orthogonal to the axis $\mathbf{m}$.

As long as $\overline{\mathbf{a}}_{12 m}$ and $\overline{\mathbf{a}}_{13 m}$ are not parallel to each other (by appropriate choices of $\theta_{1}$ and $\theta_{2}$ ), their vector product can be used to estimate the rotation axis $\mathbf{n}$, since both of them are orthogonal to $\mathbf{n}$. The vector product of $\overline{\mathbf{a}}_{12 m}$ and $\overline{\mathbf{a}}_{13 m}$ is

$$
\overline{\mathbf{a}}_{12 m} \times \overline{\mathbf{a}}_{13 m}=\left(\left(\mathbf{R}\left(\mathbf{n}, \theta_{1}\right)-\mathbf{I}\right) \mathbf{a}\right) \times\left(\left(\mathbf{R}\left(\mathbf{n}, \theta_{2}\right)-\mathbf{I}\right) \mathbf{a}\right) .
$$

Substituting (6), (11) can be rewritten as

$$
\begin{aligned}
\overline{\mathbf{a}}_{12 m} \times \overline{\mathbf{a}}_{13 m}= & \left(-\sin \theta_{1} \cdot[|\mathbf{n}|] \mathbf{a}+\left(1-\cos \theta_{1}\right)[|\mathbf{n}|]^{2} \mathbf{a}\right) \\
& \times\left(-\sin \theta_{2} \cdot \mathbf{n}[|\mathbf{n}|] \mathbf{a}+\left(1-\cos \theta_{2}\right)[|\mathbf{n}|]^{2} \mathbf{a}\right) .
\end{aligned}
$$

Using the relations $(a \mathbf{u}+b \mathbf{v}) \times(c \mathbf{u}+d \mathbf{v})=a d(\mathbf{u} \times \mathbf{v})$ $+b c(\mathbf{v} \times \mathbf{u})$ and $[|\mathbf{n}|] \mathbf{a}=-\mathbf{n} \times \mathbf{a}$, it can be further simplified as

$$
\begin{aligned}
\overline{\mathbf{a}}_{12 m} \times \overline{\mathbf{a}}_{13 m}= & \sin \theta_{1}\left(1-\cos \theta_{2}\right)((\mathbf{n} \times \mathbf{a}) \times(\mathbf{n} \times(\mathbf{n} \times \mathbf{a}))) \\
& +\sin \theta_{2}\left(1-\cos \theta_{1}\right)((\mathbf{n} \times(\mathbf{n} \times \mathbf{a})) \times(\mathbf{n} \times \mathbf{a})) .
\end{aligned}
$$

With the formula $\mathbf{u} \times(\mathbf{v} \times \mathbf{w})=(\mathbf{u} \cdot \mathbf{w}) \mathbf{v}-(\mathbf{u} \cdot \mathbf{v}) \mathbf{w}$, we have

$$
\begin{aligned}
\overline{\mathbf{a}}_{12 m} \times \overline{\mathbf{a}}_{13 m}= & \sin \theta_{1}\left(1-\cos \theta_{2}\right)((\mathbf{n} \times \mathbf{a}) \times((\mathbf{n} \cdot \mathbf{a}) \mathbf{n}-(\mathbf{n} \cdot \mathbf{n}) \mathbf{a})) \\
& +\sin \theta_{2}\left(1-\cos \theta_{1}\right)(((\mathbf{n} \cdot \mathbf{a}) \mathbf{n}-(\mathbf{n} \cdot \mathbf{n}) \mathbf{a}) \times(\mathbf{n} \times \mathbf{a})) \\
= & \left(\sin \theta_{1}\left(1-\cos \theta_{2}\right)-\sin \theta_{2}\left(1-\cos \theta_{1}\right)\right) \\
& \times\left(|\mathbf{n}|^{2} l^{2}-(\mathbf{n} \cdot \mathbf{a})^{2}\right) \mathbf{n} \\
= & \left(\sin \theta_{1}\left(1-\cos \theta_{2}\right)-\sin \theta_{2}\left(1-\cos \theta_{1}\right)\right) \\
& \times\left(l^{2}-(\mathbf{n} \cdot \mathbf{a})^{2}\right) \mathbf{n} .
\end{aligned}
$$

Hence, with the assumption of the rotation axis $\mathbf{n}$ not parallel to the baseline vector $\mathbf{a}$, the above formula can be used to check whether $\overline{\mathbf{a}}_{12 m}$ and $\overline{\mathbf{a}}_{13 m}$ are not parallel to each other. If $\overline{\mathbf{a}}_{12 m}$ and $\overline{\mathbf{a}}_{13 m}$ are not parallel to each other, the rotation axis $\mathbf{n}$ can be estimated by the formula

$$
\hat{\mathbf{n}}=\frac{\overline{\mathbf{a}}_{12 m} \times \overline{\mathbf{a}}_{13 m}}{\left|\overline{\mathbf{a}}_{12 m} \times \overline{\mathbf{a}}_{13 m}\right|}
$$

where the symbol ^ means the estimated value of the corresponding vector.

On the other hand, the rotation axis $\mathbf{m}$ can be estimated by $\overline{\mathbf{a}}_{14 m}$ and $\overline{\mathbf{a}}_{15 m}$, which are assumed not in parallel, by following similar argument as

$$
\hat{\mathbf{m}}=\frac{\overline{\mathbf{a}}_{14 m} \times \overline{\mathbf{a}}_{15 m}}{\left|\overline{\mathbf{a}}_{14 m} \times \overline{\mathbf{a}}_{15 m}\right|} .
$$

In (10), since the matrix $(\mathbf{R}(\mathbf{n}, \theta)-\mathbf{I})$ is not invertible, the pseudoinverse is used. Given a 3 by 3 real matrix $\mathbf{H}$, its singular value decomposition (SVD) [9] representation is

$$
\mathbf{H}=\mathbf{U}\left[\begin{array}{ccc}
\sigma_{1} & 0 & 0 \\
0 & \sigma_{2} & 0 \\
0 & 0 & \sigma_{3}
\end{array}\right] \mathbf{V}^{\mathrm{T}}
$$

where $\sigma_{i}(i=1,2,3)$ are singular values; $\mathbf{U}$ and $\mathbf{V}$ are orthogonal matrices whose columns are normalized right and left singular vectors, respectively. If the $\mathbf{H}$ matrix is not invertible, some singular values $\sigma_{i}(i=$ $1,2,3)$ will be zero. The generalized pseudoinverse $\mathbf{H}^{+}$ is defined as follows:

$$
H^{+}=\mathbf{V}\left[\begin{array}{ccc}
\alpha_{1} & 0 & 0 \\
0 & \alpha_{2} & 0 \\
0 & 0 & \alpha_{3}
\end{array}\right] \mathbf{U}^{\mathrm{T}}
$$

where

$$
\alpha_{i}=\left\{\begin{array}{cc}
1 / \sigma_{i}, & \sigma_{i} \neq 0 \\
0, & \sigma_{i}=0
\end{array}, \quad i=1,2,3 .\right.
$$

From the geometric viewpoint, it is obvious that the rotation axes $\mathbf{n}, \mathbf{m}$ are in the null space of the matrix $(\mathbf{R}(\mathbf{n}, \theta)-\mathbf{I}),(\mathbf{R}(\mathbf{m}, \varphi)-\mathbf{I})$, respectively. The baseline vector a can be decomposed into two orthogonal parts

$$
\mathbf{a}=\mathbf{a}_{\perp \mathbf{n}}+\mathbf{a}_{/ / \mathbf{n}}
$$

where $\mathbf{a}_{\perp \mathbf{n}}$ is orthogonal to the rotation axis $\mathbf{n}$, and $\mathbf{a}_{/ / \mathbf{n}}$ is parallel to the axis $\mathbf{n}$. Considering the singularity of $(\mathbf{R}(\mathbf{n}, \theta)-\mathbf{I})$, the generalized pseudoinverse solution of (10a) is the component $\mathbf{a}_{\perp \mathbf{n}}$. The magnitude of $\mathbf{a}_{/ / \mathbf{n}}$ can be computed by $\sqrt{l^{2}-\left|\mathbf{a}_{\perp \mathbf{n}}\right|^{2}}$, but the direction of $\mathbf{a}_{/ / \mathbf{n}}$ cannot be determined yet. In fact, from $\overline{\mathbf{a}}_{12 m}$ and $\overline{\mathbf{a}}_{13 m}$, there are two possible solutions for $\mathbf{a}_{\perp \mathbf{n}}$. In order to accommodate some perturbations in the measurements, a weighted average for estimating $\mathbf{a}_{\perp \mathbf{n}}$ is adopted as follows,

$$
\begin{array}{r}
\hat{\mathbf{a}}_{\perp \mathbf{n}}=\alpha\left(\mathbf{R}\left(\hat{\mathbf{n}}, \theta_{1}\right)-\mathbf{I}\right)^{+} \overline{\mathbf{a}}_{12 m}+(1-\alpha)\left(\mathbf{R}\left(\hat{\mathbf{n}}, \theta_{2}\right)-\mathbf{I}\right)^{+} \overline{\mathbf{a}}_{13 m}, \\
0 \leq \alpha \leq 1
\end{array}
$$

where the coefficient $\alpha$ is determined according to the accuracy of the measurements for $\overline{\mathbf{a}}_{12 m}$ and $\overline{\mathbf{a}}_{13 m}$. If $\overline{\mathbf{a}}_{12 m}$ and $\overline{\mathbf{a}}_{13 m}$ have similar error characteristics, the 
value of $\alpha$ is typically chosen as 0.5 . Similarly, the baseline vector a can also be decomposed as

$$
\mathbf{a}=\mathbf{a}_{\perp \mathbf{m}}+\mathbf{a}_{/ / \mathbf{m}}
$$

where $\mathbf{a}_{\perp \mathbf{m}}$ can be estimated as

$$
\begin{aligned}
& \hat{\mathbf{a}}_{\perp \mathbf{m}}= \beta\left(\mathbf{R}\left(\hat{\mathbf{m}}, \varphi_{1}\right)-\mathbf{I}\right)^{+} \overline{\mathbf{a}}_{14 m} \\
&+(1-\beta)\left(\mathbf{R}\left(\hat{\mathbf{m}}, \varphi_{2}\right)-\mathbf{I}\right)^{+} \overline{\mathbf{a}}_{15 m}, \\
& 0 \leq \beta \leq 1
\end{aligned}
$$

The magnitude of $\hat{\mathbf{a}}_{/ / \mathbf{m}}$ is $\sqrt{l^{2}-\left|\hat{\mathbf{a}}_{\perp \mathbf{m}}\right|^{2}}$; the direction of $\hat{\mathbf{a}}_{/ \mathbf{m}}$ is unknown yet.

By the properties of the pseudoinverse, the vectors $\hat{\mathbf{a}}_{\perp \mathbf{n}}$ and $\hat{\mathbf{a}}_{\perp \mathbf{m}}$ computed in (15) and (17), must be orthogonal to the estimated rotation axes $\hat{\mathbf{n}}, \hat{\mathbf{m}}$, respectively.

From either (14) or (16), the desired a can be estimated if the direction of $\mathbf{a}_{/ / \mathbf{n}}$ or $\mathbf{a}_{/ / \mathbf{m}}$ is known. The possible estimates of $\mathbf{a}$ are then

$$
\begin{aligned}
& \xi_{1}=\hat{\mathbf{a}}_{\perp \mathbf{n}}+\sqrt{l^{2}-\left|\hat{\mathbf{a}}_{\perp \mathbf{n}}\right|^{2}} \hat{\mathbf{n}} \\
& \xi_{2}=\hat{\mathbf{a}}_{\perp \mathbf{n}}-\sqrt{l^{2}-\left|\hat{\mathbf{a}}_{\perp \mathbf{n}}\right|^{2}} \hat{\mathbf{n}} \\
& \xi_{3}=\hat{\mathbf{a}}_{\perp \mathbf{m}}+\sqrt{l^{2}-\left|\hat{\mathbf{a}}_{\perp \mathbf{m}}\right|^{2}} \hat{\mathbf{m}} \\
& \xi_{4}=\hat{\mathbf{a}}_{\perp \mathbf{m}}-\sqrt{l^{2}-\left|\hat{\mathbf{a}}_{\perp \mathbf{m}}\right|^{2}} \hat{\mathbf{m}}
\end{aligned}
$$

where $\hat{\mathbf{a}}_{\perp \mathbf{n}}, \hat{\mathbf{a}}_{\perp \mathbf{m}}$ are computed from (15), (17). The problem next is to determine which $\xi_{i}$ is the appropriate estimate for $\mathbf{a}$. There are four possibilities to be considered.

1) $\sqrt{l^{2}-\left|\hat{\mathbf{a}}_{\perp \mathbf{n}}\right|^{2}} \cong 0$. Then $\xi_{1} \cong \xi_{2}$, and if one of $\xi_{3}$, $\xi_{4}$ is very close to $\xi_{1}$, the desired estimate is $\hat{\mathbf{a}}=\xi_{1}$. Otherwise, there is no appropriate solution.

2) $\sqrt{l^{2}-\left|\hat{\mathbf{a}}_{\perp \mathbf{m}}\right|^{2}} \cong 0$. Then $\xi_{3} \cong \xi_{4}$, and if one of $\xi_{1}, \xi_{2}$ is very close to $\xi_{3}$, the desired estimate is $\hat{\mathbf{a}}=\xi_{3}$. Otherwise, no appropriate solution exists.

3) $\sqrt{l^{2}-\left|\hat{\mathbf{a}}_{\perp \mathbf{n}}\right|^{2}} \cong 0 \cong \sqrt{l^{2}-\left|\hat{\mathbf{a}}_{\perp \mathbf{m}}\right|^{2}}$. Then $\xi_{1} \cong \xi_{2}$, $\xi_{3} \cong \xi_{4}$. If $\xi_{1} \cong \xi_{3}$, then the solution is $\xi_{1}$. Otherwise, there is no solution.

4) $\sqrt{l^{2}-\left|\hat{\mathbf{a}}_{\perp \mathbf{n}}\right|^{2}} \neq 0, \sqrt{l^{2}-\left|\hat{\mathbf{a}}_{\perp \mathbf{m}}\right|^{2}} \neq 0$. Then $\xi_{1} \neq \xi_{2}$ and $\xi_{3} \neq \xi_{4}$. If the solution exists, one of $\xi_{1}$, $\xi_{2}$ must be very close to one of $\xi_{3}, \xi_{4}$, which is the desired estimate. Otherwise, no appropriate solution exists.

Based on the above discussions, the general algorithm to estimate the baseline vector $\mathbf{a}$ and the rotation axes $\mathbf{n}, \mathbf{m}$ is listed as follows.

\section{Algorithm 1.}

Given an unknown baseline vector a, with known length $l$, which can be rotated about the axes $\mathbf{n}$ and $\mathbf{m}$, respectively. The baseline vector a and rotation axes $\mathbf{n}$ and $\mathbf{m}$ can be estimated through the following procedure.
1) Acquire necessary measurements by a) rotating a about the axis $\mathbf{n}$ twice, b) turning back, and c) rotating a about the axis $\mathbf{m}$ twice.

2) Compute $\mathbf{a}_{i m}, i=1, \ldots, 5$ via (8), with the double differences of the phase observables (7).

3) Compute $\mathbf{a}_{12 m}$ to $\mathbf{a}_{15 m}$ by differences between $\mathbf{a}_{i m}, i=1, \ldots, 5$ via (9), and perform averaging.

4) Use (12) and (13) to compute $\hat{\mathbf{n}}$ and $\hat{\mathbf{m}}$.

5) Find $\hat{\mathbf{a}}_{\perp \mathbf{n}}$ and $\hat{\mathbf{a}}_{\perp \mathbf{m}}$ by (15) and (17).

6) Compute four possible candidates for the baseline vector a by (18).

7) Choose the appropriate solution by considering four possibilities.

Based on the estimates of the baseline vector a and two rotation axes $\mathbf{n}, \mathbf{m}$, the double-difference integer ambiguities can be computed by

$$
\begin{gathered}
\hat{\mathbf{N}}=\operatorname{int}\left(\frac { 1 } { 5 \lambda } \left(\Phi_{1}-\mathbf{G}^{\mathrm{T}} \hat{\mathbf{a}}+\sum_{i=2}^{3}\left(\Phi_{i}-\mathbf{G}^{\mathrm{T}} \mathbf{R}\left(\hat{\mathbf{n}}, \theta_{i-1}\right) \hat{\mathbf{a}}\right)\right.\right. \\
\left.+\sum_{i=4}^{5}\left(\Phi_{i}-\mathbf{G}^{\mathrm{T}} \mathbf{R}\left(\hat{\mathbf{m}}, \varphi_{i-3}\right) \hat{\mathbf{a}}\right)\right)
\end{gathered}
$$

where int( $\cdot$ ) means rounding to the nearest integer. Since the integer ambiguities are determined, they can be used in the subsequent measurements to calculate the direction of the baseline vectors.

One application of Algorithm 1 is to determine the line of sight (LOS) of a tracking radar. The normal plane of the LOS is formed by the perimeter of radar's disk antenna. If three GPS antennas are mounted at three points of the rim of the radar's antenna, say points $A, B$, and $C$, then the cross product of the vectors $\mathbf{a}_{A B}$ and $\mathbf{a}_{B C}$ will coincide with the LOS of this radar. Considering the antenna pedestal, the azimuth and the elevation turning mechanisms already exist. The rotation axes $\mathbf{n}$ and $\mathbf{m}$ in Algorithm 1 are the radar antenna's azimuth and elevation axes, respectively. To determine $\mathbf{a}_{A B}$ and $\mathbf{a}_{B C}$, two azimuth rotations and two elevation rotations are needed as described in this section.

In Algorithm 1, there is no specific assumptions on $\mathbf{n}$ and $\mathbf{m}$, and their relations with $\mathbf{a}$, except that they cannot be parallel to each other. If there are some relationships between $\mathbf{n}, \mathbf{m}$, and $\mathbf{a}$, the algorithm may be simplified significantly. For certain applications in which such relationships exist, the problem of direction finding can be solved much more easily.

\section{SIMPLIFIED BASELINE ROTATION METHOD}

The direction finding is a critical issue for artillery rockets. If two GPS antennas are fixed at both ends of the launch pot container (LPC), then the formed baseline will match the aiming direction of the rocket. Please note that for such armaments, the azimuth and the elevation turning mechanisms are well equipped already. Furthermore, the azimuth axis and the 


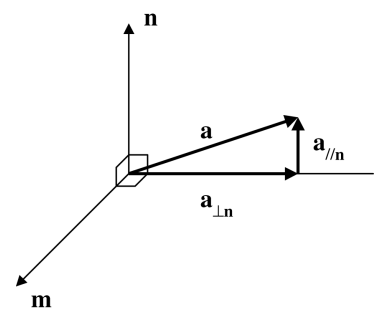

Fig. 3. Simplified configuration of two-axis platform with $\mathbf{m}$ and n orthogonal, $\mathbf{m}$ and a orthogonal.

elevation axis are orthogonal to each other and the baseline vector (LPC) is orthogonal to the elevation axis. In our framework established in Section III, the rotation axes $\mathbf{n}$ and $\mathbf{m}$ represent the azimuth axis and the elevation axis, respectively. The baseline vector a built by two GPS antennas will stand for the aiming direction of the artillery rocket. Because more information about $\mathbf{a}, \mathbf{m}$, and $\mathbf{n}$ has been revealed, the algorithm for direction finding should be simplified. Later we show that two rotations about $\mathbf{n}$ (azimuth) axis and one rotation about $\mathbf{m}$ (elevation) axis are enough for direction finding.

As shown in Fig. 3, $\mathbf{m} \cdot \mathbf{n}=0$ and $\mathbf{m} \cdot \mathbf{a}=0$, and

$$
\mathbf{a}=\mathbf{a}_{\perp \mathbf{n}}+\mathbf{a}_{/ / \mathbf{n}} .
$$

It is noted that the three vectors $\mathbf{m}, \mathbf{n}, \mathbf{a}_{\perp \mathbf{n}}$ are orthogonal to each other and can form a triad. Accordingly, the vector $\mathbf{m}$ can be directly obtained by the formula

$$
\hat{\mathbf{m}}=\frac{\hat{\mathbf{a}}_{\perp \mathbf{n}} \times \hat{\mathbf{n}}}{\left|\hat{\mathbf{a}}_{\perp \mathbf{n}} \times \hat{\mathbf{n}}\right|}
$$

once $\hat{\mathbf{n}}$ and $\hat{\mathbf{a}}_{\perp \mathbf{n}}$ are computed. It is thus not necessary to find $\hat{\mathbf{a}}_{\perp \mathbf{m}}$ in Algorithm 1. However, a rotation about the axis $\mathbf{m}$ is still necessary to determine the sign of $\hat{\mathbf{a}}_{/ / \mathbf{n}}$ in the algorithm.

Following similar notations in the previous section, two rotations of the baseline vector a about the axis $\mathbf{n}$ by the two given angles $\theta_{1}, \theta_{2}$ and one rotation about the axis $\mathbf{m}$ by the given angle $\varphi$, are performed. Instead of $(5 \mathrm{c})$, we have

$$
\mathbf{a}_{4}=\mathbf{R}(\mathbf{m}, \varphi) \mathbf{a}
$$

and the corresponding phase observables

$$
\begin{aligned}
\Phi_{4} & =\mathbf{G}^{\mathrm{T}} \mathbf{a}_{4}+\lambda \mathbf{N}+\mathbf{B}+\mathbf{W}_{4} \\
& =\mathbf{G}^{\mathrm{T}} \mathbf{R}(\mathbf{m}, \varphi) \mathbf{a}+\lambda \mathbf{N}+\mathbf{B}+\mathbf{W}_{4} .
\end{aligned}
$$

With $\hat{n}, \hat{\mathbf{a}}_{\perp \mathbf{n}}$ computed in the same way as in Algorithm 1, and $\hat{\mathbf{m}}$ estimated by (20), the baseline vector a can be estimated by

$$
\hat{\mathbf{a}}^{\prime}=(\mathbf{R}(\hat{\mathbf{m}}, \varphi)-\mathbf{I})^{+} \overline{\mathbf{a}}_{14 m}
$$

where $\overline{\mathbf{a}}_{14 m}$ is the sample average on the result of $\mathbf{a}_{14 m}$ in (9b). On the other hand, the vectors $\xi_{1}, \xi_{2}$ are computed by (18a), (18b), respectively. If $\hat{\mathbf{a}}^{\prime}$ is close to $\xi_{1}$, then the desired estimation is $\xi_{1}$. If $\hat{\mathbf{a}}^{\prime}$ is close to $\xi_{2}$, then it is the solution. Otherwise, no appropriate solution exists.

The above discussion is summarized by the following algorithm.

Algorithm 2.

1) Acquire necessary measurements by a) rotating $\mathbf{a}$ about the axis $\mathbf{n}$ twice, $b$ ) turning back, and c) rotating $\mathbf{a}$ about the axis $\mathbf{m}$ once.

2) Compute $\mathbf{a}_{i m}, i=1, \ldots, 4$ via (8), with the double differences of the phase observables in (7a), (7b), (22).

3) Compute $\mathbf{a}_{12 m}$ to $\mathbf{a}_{14 m}$ by differences between $\mathbf{a}_{i m}, i=1, \ldots, 4$ via (9), and perform averaging.

4) Use (12) to compute $\hat{\mathbf{n}}$.

5) Find $\hat{\mathbf{a}}_{\perp \mathbf{n}}$ by (15).

6) Estimate the axis $\mathbf{m}$ by (20).

7) Compute $\hat{\mathbf{a}}^{\prime}$ by (23).

8) Compute $\xi_{1}, \xi_{2}$ by (18a), (18b). If $\xi_{1} \cong \hat{\mathbf{a}}^{\prime}$, then $\hat{\mathbf{a}}=\xi_{1}$. If $\xi_{2}=\hat{\mathbf{a}}^{\prime}$, then $\hat{\mathbf{a}}=\xi_{2}$. Otherwise, there is no solution.

The direction finding problems could be degenerated to the circumstance of one-axis platform. For example, to determine the heading vector of a vehicle, we may put a rotational mechanism with one bar aligned with the direction of the vehicle as the baseline vector. The bar can be turned about a rotation axis, which is orthogonal to the bar. Comparing with the general case of Section III, this circumstance corresponds to the setting that the baseline vector a and the rotation axis $\mathbf{n}$ are orthogonal to each other (i.e., $\mathbf{a} \cdot \mathbf{n}=0$ ). The algorithm used in the general case can then be simplified significantly. Only two rotations about the axis $\mathbf{n}$ are adequate for direction finding.

Following similar notations in the Section III, two rotations of the baseline vector $\mathbf{a}$ about the axis $\mathbf{n}$ by the two given angles $\theta_{1}, \theta_{2}$ are performed. Then $\hat{\mathbf{n}}$, $\hat{\mathbf{a}}_{\perp \mathbf{n}}$ are computed in the same way as in Algorithm 1. Since the baseline vector $\mathbf{a}$ is orthogonal to the rotation axis $\mathbf{n}, \hat{\mathbf{a}}_{/ / \mathbf{n}}$ is zero, hence, $\hat{\mathbf{a}}_{\perp \mathbf{n}}$ is the estimate of $\mathbf{a}$.

The above discussion is summarized by the following algorithm.

Algorithm 3.

1) Acquire necessary measurements by rotating a about $\mathbf{n}$ twice.

2) Compute $\mathbf{a}_{i m}, i=1, \ldots, 3$ via (8), with the double differences of the phase observables in (7a), (7b)

3) Compute $\mathbf{a}_{12 m}$ and $\mathbf{a}_{13 m}$ by differences between $\mathbf{a}_{i m}, i=1,2,3$ via (9a), and perform averaging.

4) Use (12) to compute $\hat{\mathbf{n}}$.

5) Find $\hat{\mathbf{a}}_{\perp \mathbf{n}}$ by (15). If $l \cong\left|\hat{\mathbf{a}}_{\perp \mathbf{n}}\right|$, then $\hat{\mathbf{a}}=\hat{\mathbf{a}}_{\perp \mathbf{n}}$. Otherwise, no appropriate solution exists.

In fact, the results of [8] are equivalent to those Algorithm 3. 
The assumptions of Algorithm 2 (namely, a) the baseline vector (a) perpendicular to one rotation axis $(\mathbf{m})$, and b) two rotation axes ( $\mathbf{m}$ and $\mathbf{n}$ ) perpendicular to each other) are more stringent than those of Algorithm 3 (i.e., the baseline vector (a) perpendicular to the rotation axis (n)). From the mathematical point of view, one can apply Algorithm 3 to solve the direction finding problem in the environments satisfying assumptions of Algorithm 2. That is, just rotate the baseline about the axis $\mathbf{m}$ twice, then use Algorithm 3 to compute a. But when we consider the real world situations, sometimes the restrictions on the rotation angles about $\mathbf{m}$ and $\mathbf{n}$ are quite different. Taking the artillery rocket in the circumstance of Algorithm 2 as an example, we explain why Algorithm 2 is still an appropriate choice. The azimuth rotation axis of the LPC is equivalent to $\mathbf{n}$. This axis does not need to be perpendicular to the direction vector (a), which coincides with the direction of LPC, since the launcher might be inclined but the azimuth rotation axis is straight all the time. The elevation rotation axis of the LPC is equivalent to $\mathbf{m}$. This axis is always perpendicular to a. If we apply Algorithm 2, the LPC is rotated about the azimuth axis (n) twice and the elevation axis (m) once. If we apply Algorithm 3, the LPC is rotated about the elevation axis $(\mathbf{m})$ twice. The extents of rotation angles about the azimuth axis and the elevation axis depend on the specifications of individual artillery rocket. However, in general cases the ranges of elevation rotations are more restrictive than those of azimuth rotations. Furthermore, the GPS antenna mounted on a highly inclined LPC will be difficult to receive all satellite signals coming from the whole sky. The limited elevation rotation angles will cause more serious errors if Algorithm 3 is used. On the contrary, the two rotations about the azimuth axis are less restrictive. Hence, Algorithm 2 has the chance to be used to obtain the more accurate results.

Similar to (19), the double-difference integer ambiguities can be found, by the estimate of the baseline vector, as

$$
\begin{gathered}
\hat{\mathbf{N}}=\operatorname{int}\left(\frac { 1 } { 4 \lambda } \left(\Phi_{1}-\mathbf{G}^{\mathrm{T}} \hat{\mathbf{a}}+\sum_{i=2}^{3}\left(\Phi_{i}-\mathbf{G}^{\mathrm{T}} \mathbf{R}\left(\hat{\mathbf{n}}, \theta_{i-1}\right) \hat{\mathbf{a}}\right)\right.\right. \\
\left.\left.+\Phi_{4}-\mathbf{G}^{\mathrm{T}} \mathbf{R}(\hat{\mathbf{m}}, \varphi) \hat{\mathbf{a}}\right)\right) \\
\hat{\mathbf{N}}=\operatorname{int}\left(\frac{1}{3 \lambda}\left(\Phi_{1}-\mathbf{G}^{\mathrm{T}} \hat{\mathbf{a}}+\sum_{i=2}^{3}\left(\Phi_{i}-\mathbf{G}^{\mathrm{T}} \mathbf{R}\left(\hat{\mathbf{n}}, \theta_{i-1}\right) \hat{\mathbf{a}}\right)\right)\right.
\end{gathered}
$$

for the above two cases, respectively.

\section{THE EXPERIMENT}

In order to verify the validity of the algorithms discussed, an experiment was performed. Based on

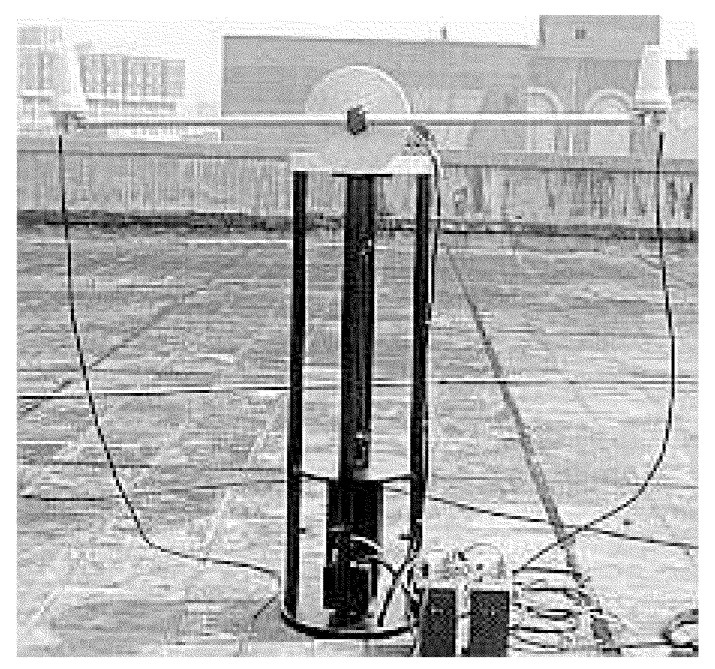

Fig. 4. Experimental equipment configuration.

the assumptions of the simplified case described in Section IV, a two-degree-of-freedom platform has been set up. The configuration of the equipment is shown in Fig. 4, for which Algorithm 2 can be applied.

To assess the effects of different parameters in the experiment, the simulations were conducted first based on the above configuration. The simulation results helped us to choose the experimental parameters, such as the length of the baseline and the rotation angles, etc. In the simulations, the white noises $\mathcal{N}\left(0,(0.01 \lambda)^{2}\right)$ were added in phase measurements to evaluate the effects caused by the changes of the baseline length, the rotation angles, the initial baseline azimuth, or the elevation angles. Four GPS satellites were used in the simulations in which one satellite was in the zenith and the other three satellites were 120 deg angles apart in the horizon.

First, we considered the baseline vector with both the azimuth angle and the elevation angle being $45^{\circ}$. The rotation axes $\mathbf{n}$ and $\mathbf{m}$ were chosen as $\left[\begin{array}{lll}0 & 0 & 1\end{array}\right]^{\mathrm{T}}$ and $\left[\cos \left(-45^{\circ}\right) \sin \left(-45^{\circ}\right) 0\right]^{\mathrm{T}}$, respectively, in the local frame. With added noises, the carrier phase measurements were computed during the process of rotating the baseline vector about $\mathbf{n}$ to $\theta_{1}=60^{\circ}$ and to $\theta_{2}=-60^{\circ}$, and then rotating the baseline vector about m by $\varphi=30^{\circ}$. With the generated phase observables, Algorithm 2 was applied to find the direction of the baseline vector for the different length varying from $0.5 \mathrm{~m}$ to $2 \mathrm{~m}$ with the step size $0.1 \mathrm{~m}$. For every baseline length, one thousand times of carrier phases were generated. The sample standard deviations for the azimuth angle and the elevation angle of the baseline vector are shown in Figs. 5 and 6. It could be concluded that with larger baseline lengths, the errors are reduced. This matches with the observation that the errors of relative positioning do not vary significantly with respect to different baseline lengths, 


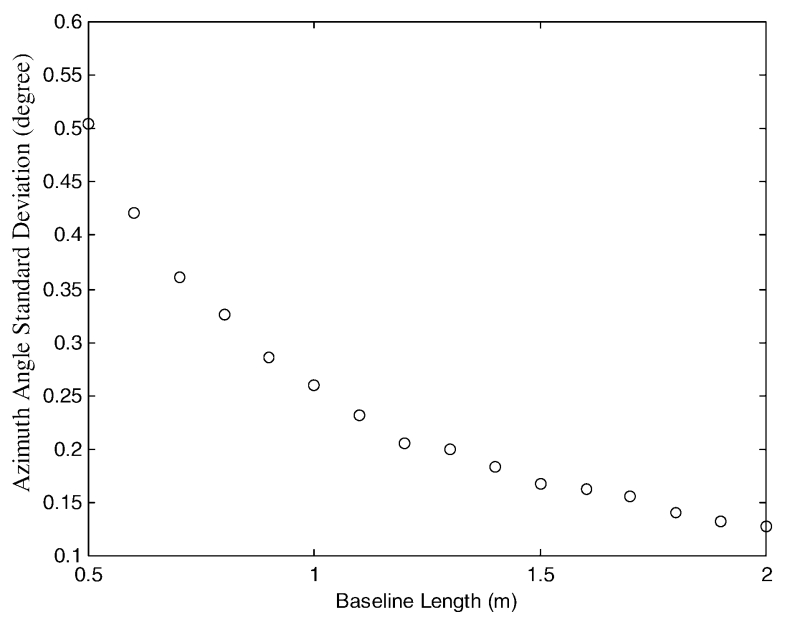

Fig. 5. Simulation 1: azimuth errors at different baseline lengths.

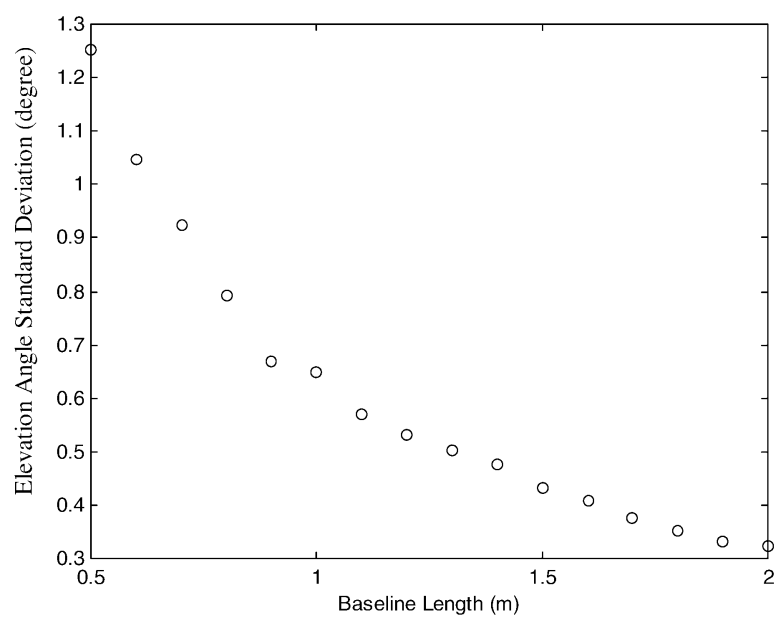

Fig. 6. Simulation 1: elevation errors at different baseline lengths.

and thus the errors of the angles estimated must be smaller for longer baseline.

In the second simulation, the length of the baseline vector was chosen as $1.2 \mathrm{~m}$. The setting is similar to the one in the first simulation, except that the different rotation angle $\theta_{1}$ changing from $5^{\circ}$ to $175^{\circ}$ with the step size $5^{\circ}$ about the axis $\mathbf{n}$ are tested, while the other rotation angle $\theta_{2}$ about the axis $\mathbf{n}$ are set to be equal to $-\theta_{1}$. The rotation angle $\varphi$ about the axis $\mathbf{m}$ is still $30^{\circ}$. One thousand tests for every rotation angle $\theta_{1}$ were performed, and the sample standard deviations for the azimuth angle and the elevation angle are shown in Figs. 7 and 8. It is observed from Fig. 7 that the error for the azimuth angle attains the minimum value when the rotation angle was about $90^{\circ}$. On the other hand, Fig. 8 shows that the error for the elevation angle is decreasing, as the rotation angle becomes larger.

To determine the best configuration in the algorithm, the simulation was performed corresponding to different azimuth angles and elevation angles of the baseline vector. The rotation

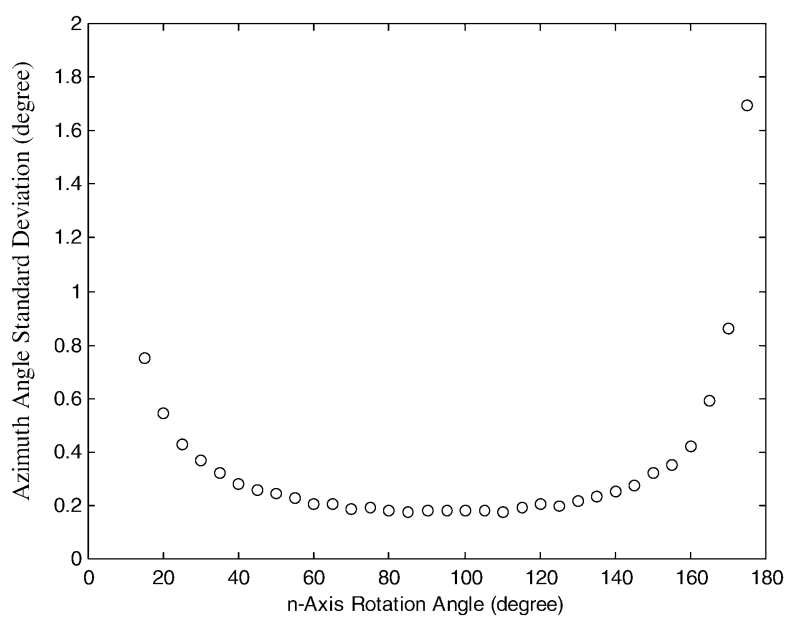

Fig. 7. Simulation 2: azimuth errors at different rotation angles.

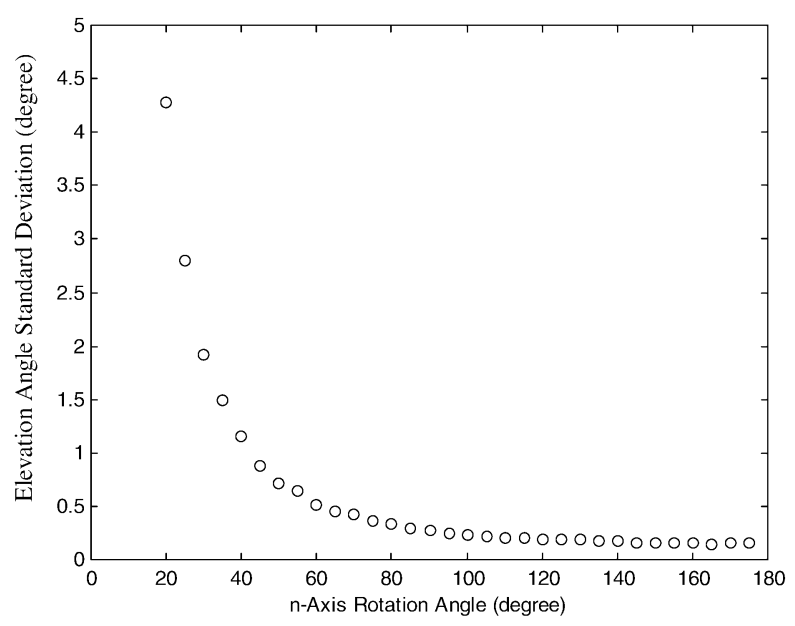

Fig. 8. Simulation 2: elevation errors at different rotation angles.

axis $\mathbf{n}$ was $\left[\begin{array}{lll}0 & 0 & 1\end{array}\right]^{\mathrm{T}}$ and the rotation angles were chosen as $\theta_{1}=60^{\circ}, \theta_{2}=-60^{\circ}$, and $\varphi=30^{\circ}$. With respect to the azimuth angle varying from $5^{\circ}$ to $175^{\circ}$, while keeping the elevation angle being $45^{\circ}$, the sample standard deviations for the azimuth angle and the elevation angle are shown in Figs. 9 and 10. On the other hand, the results for changing the elevation angles from $5^{\circ}$ to $85^{\circ}$, while keeping the azimuth angle being $45^{\circ}$ are shown in Figs. 11 and 12. As expected, the sample standard deviations of the estimated angles do not vary significantly for different azimuth angles of the baseline vectors, and the errors are larger as the elevation angles become larger.

The above simulations show that the algorithm is valid, which is further verified by the following experiment. The procedure of the experiment is described below. A $1.35 \mathrm{~m}$ aluminum bar with two antennas mounted on the both ends was used to represent the baseline vector. The GPS receivers used in our experiment are Motorola ONCORE. The first phase observables and ephemeris measurements were first taken before the baseline rotating. Then, the baseline vector was rotated about the axis $\mathbf{n}$ by 


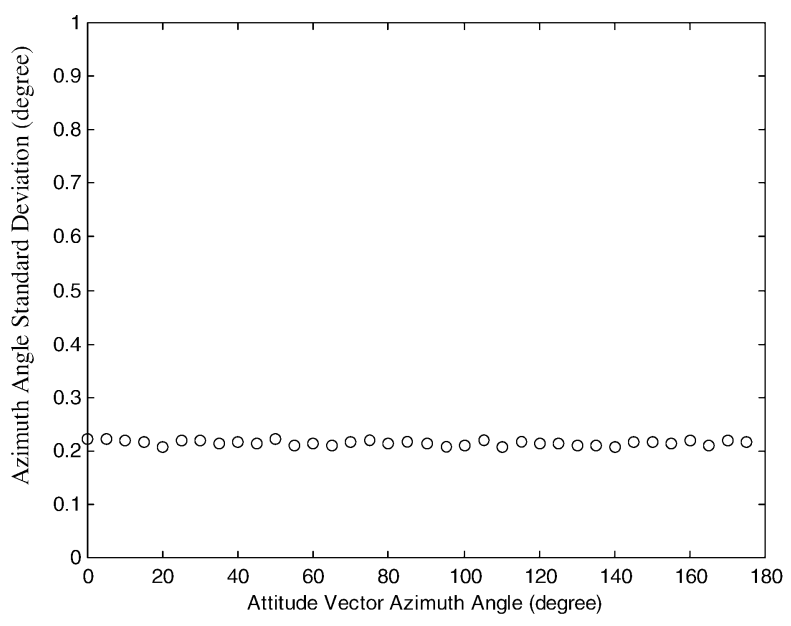

Fig. 9. Simulation 3a: azimuth errors at different azimuth angles.

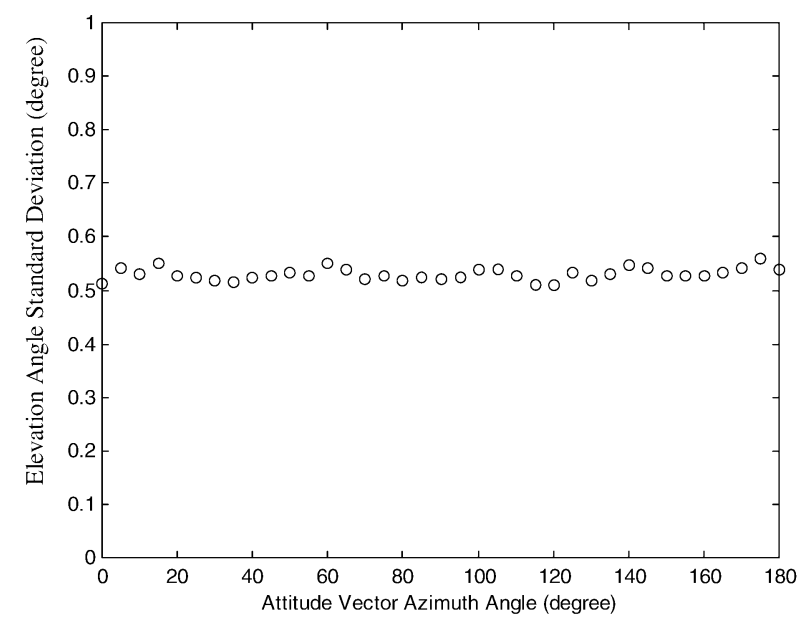

Fig. 10. Simulation 3a: elevation errors at different azimuth angles.

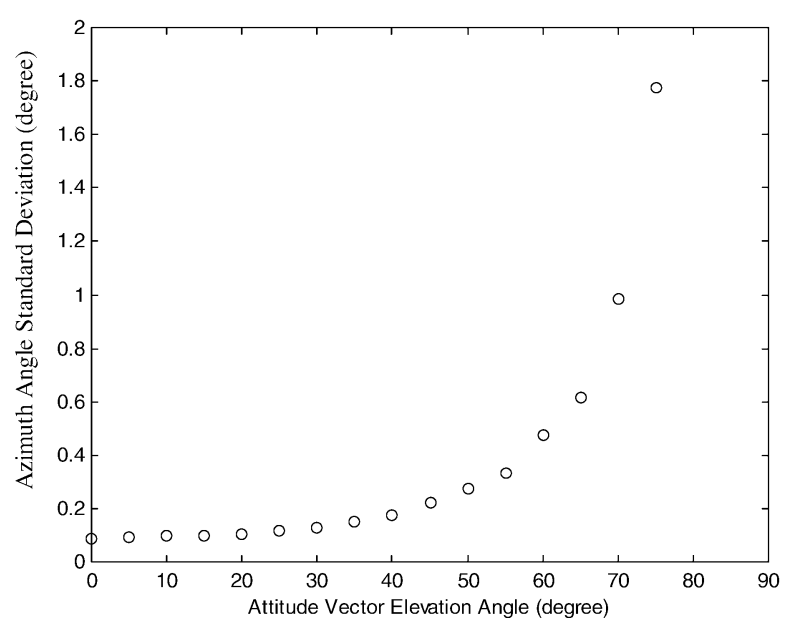

Fig. 11. Simulation 3b: azimuth errors at different elevation angles.

$60^{\circ}$, and the second phase observables were taken. Next, the bar was rotated about the axis $\mathbf{n}$ by $-120^{\circ}$, which corresponds to the vector of rotating the initial baseline vector by $-60^{\circ}$. The third measurements

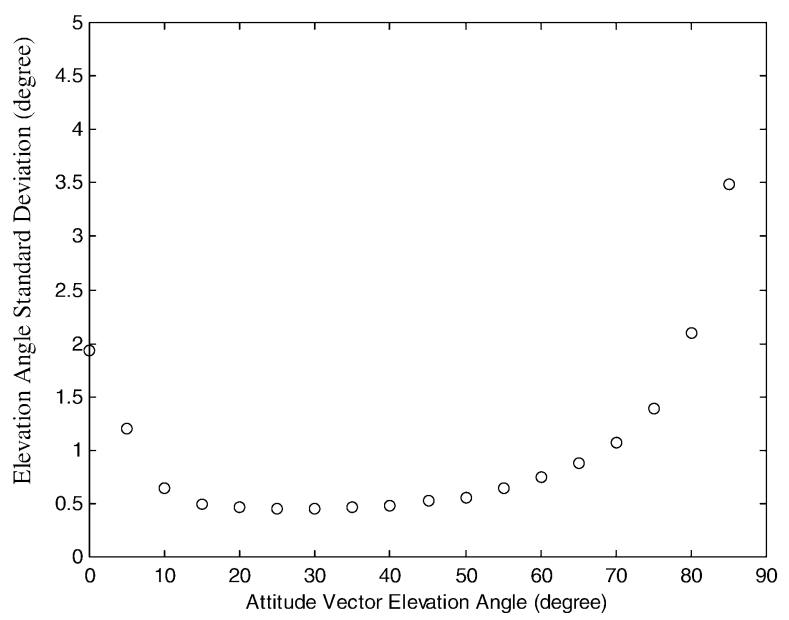

Fig. 12. Simulation 3b: elevation errors at different elevation angles.

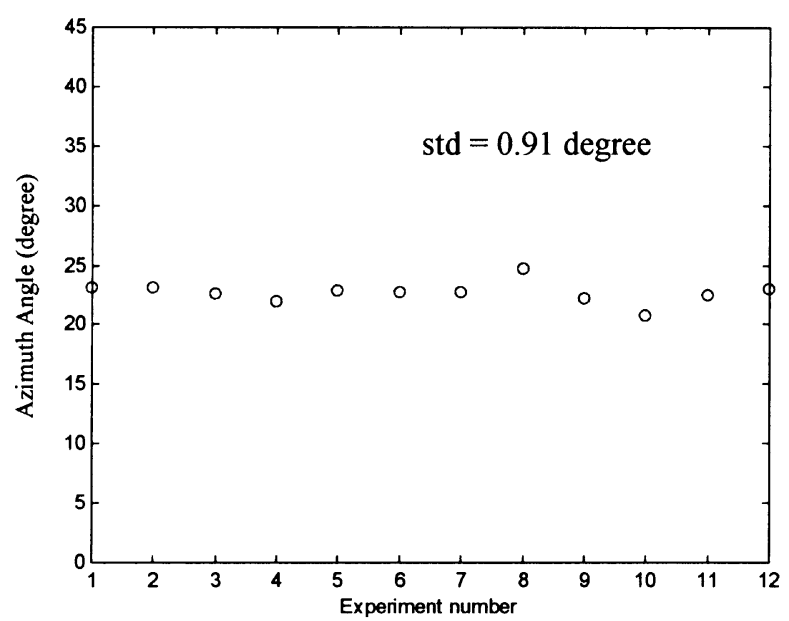

Fig. 13. Experimental results: baseline vector azimuth angle.

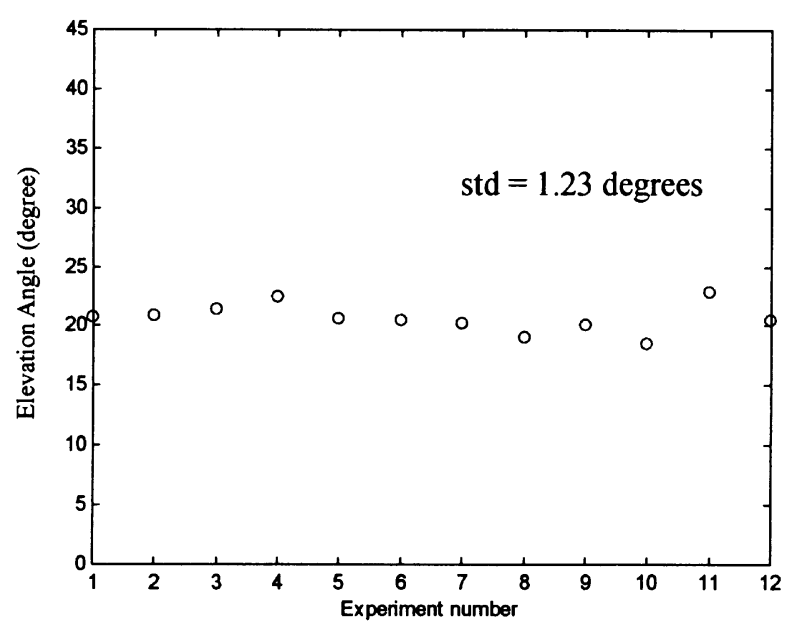

Fig. 14. Experimental results: baseline vector elevation angle.

were obtained. Finally, the bar was rotated to the initial configuration and then rotated about the axis $\mathbf{m}$ by $30^{\circ}$, in which the fourth observables were measured. 


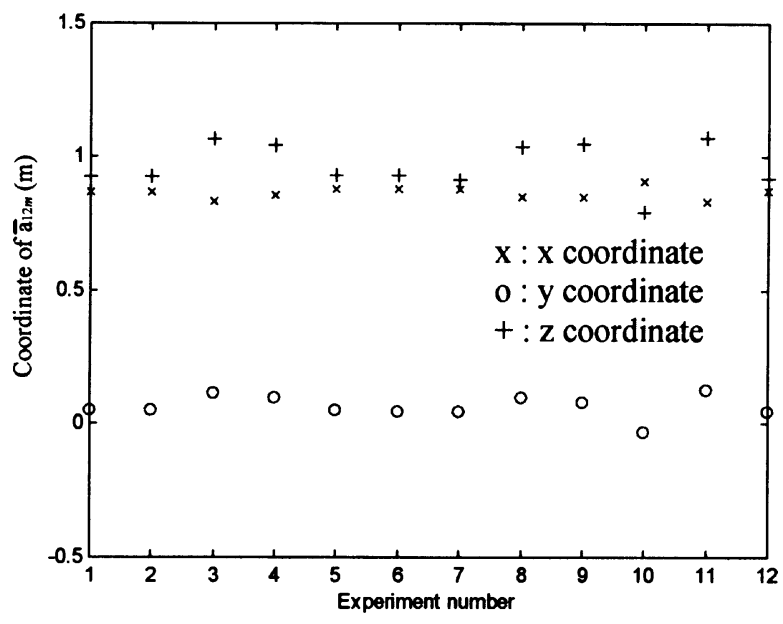

Fig. 15. Experimental results: computed $\overline{\mathbf{a}}_{12 m}$.

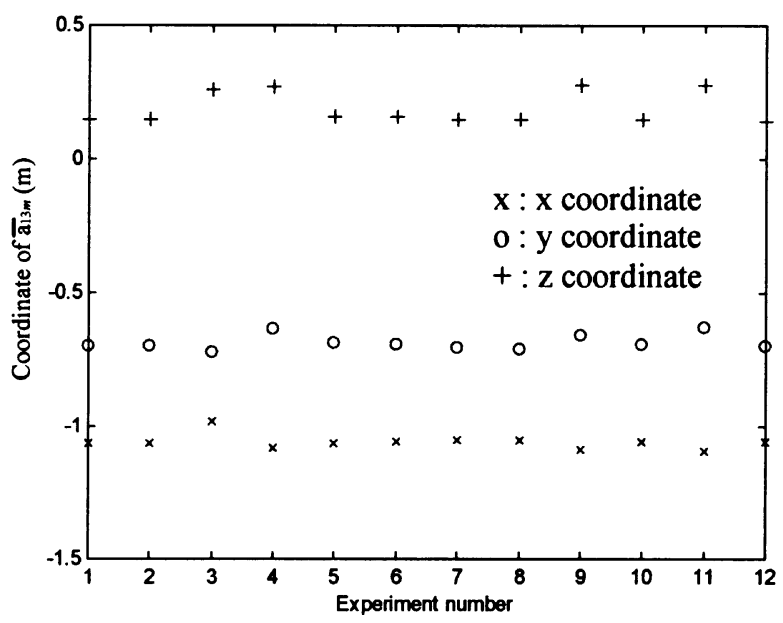

Fig. 16. Experimental results: computed $\overline{\mathbf{a}}_{13 m}$.

Algorithm 2 was then applied to find the direction of the bar. The signals from eight satellites with PRN number 1, 6, 14, 21, 22, 25, 29, 30 were used. The same procedure was repeated 12 times, with results shown in Figs. 13 and 14. The sample standard deviations for the estimated azimuth angle and the estimated elevation angle were $0.91 \mathrm{deg}$ and $1.23 \mathrm{deg}$, respectively. With the same parameters in the experiment, the simulation result shows that the sample standard deviations for the azimuth angle and the elevation angle are $0.18 \mathrm{deg}$ and $0.41 \mathrm{deg}$, respectively. The differences between the simulation and the experimental result should be induced by the unmodeled errors, such as the inaccuracy of the rotation mechanisms and the disturbance of the environments, such as the effects from the wind and the multipath. According to the observations in the experiment, the major discrepancy was distributed form environmental disturbances. Figs. 15-17 show the computed $\overline{\mathbf{a}}_{12 m}, \overline{\mathbf{a}}_{13 m}$, and $\overline{\mathbf{a}}_{14 m}$, respectively. It is noted that the accuracy can be enhanced with longer baseline. The experimental results demonstrate

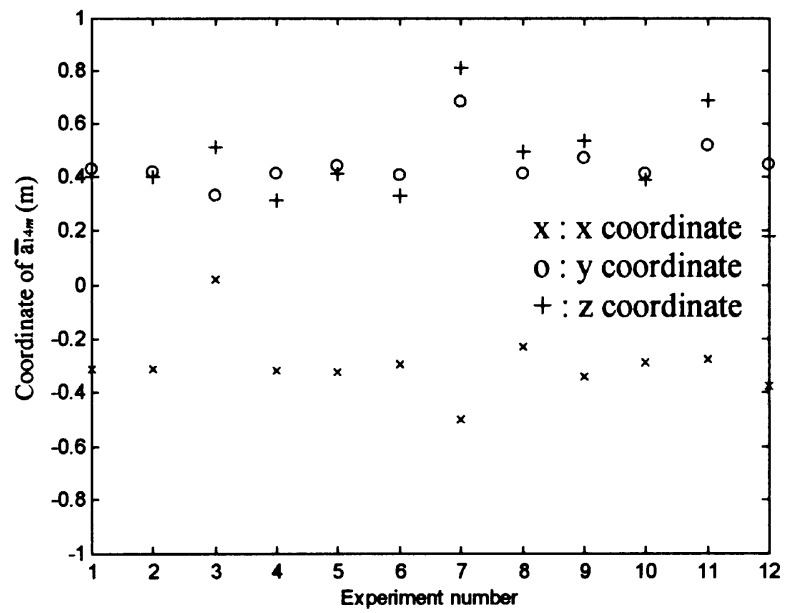

Fig. 17. Experimental results: computed $\overline{\mathbf{a}}_{14 m}$.

that the algorithm is sound, and can be used to find the direction of the baseline vector with acceptable accuracy.

\section{ERROR ANALYSIS}

Due to the mechanical problems, the rotation of the baseline vector may not be very precise. The propagation of such rotation errors is analyzed in this section. Since the effects caused by rotation errors about the axes $\mathbf{n}$ and $\mathbf{m}$, respectively, are similar, the following discussions are focused on the rotation about the axis $\mathbf{n}$. Accordingly, the error analysis for Algorithm 3 is conducted first.

Let the errors of rotation angles $\theta_{1}$ and $\theta_{2}$ be $\tilde{\theta}_{1}$ and $\tilde{\theta}_{2}$, respectively. With these rotation errors, the estimated baseline vector should be

$$
\begin{aligned}
\hat{\mathbf{a}}_{\perp \mathbf{n}}= & \alpha\left(\mathbf{R}\left(\hat{\mathbf{n}}, \theta_{1}+\tilde{\theta}_{1}\right)-\mathbf{I}\right)^{+} \overline{\mathbf{a}}_{12 m} \\
& +(1-\alpha)\left(\mathbf{R}\left(\hat{\mathbf{n}}, \theta_{2}+\tilde{\theta}_{2}\right)-\mathbf{I}\right)^{+} \overline{\mathbf{a}}_{13 m}
\end{aligned}
$$

instead of (15). Note that both $\overline{\mathbf{a}}_{12 m}$ and $\overline{\mathbf{a}}_{13 m}$ are orthogonal to the estimated rotation axis $\hat{\mathbf{n}}$. Note also the null space of $(\mathbf{R}(\hat{\mathbf{n}}, \theta)-\mathbf{I})^{\mathrm{T}}$, for $0<\theta<2 \pi$, is the set of all linear combinations of $\hat{n}$. Hence $\overline{\mathbf{a}}_{12 m}$ and $\overline{\mathbf{a}}_{13 m}$ are in the range space of $\mathbf{R}(\hat{\mathbf{n}}, \theta)-\mathbf{I}$, for $0<\theta<2 \pi$. Accordingly, there exist $\hat{\boldsymbol{\eta}}_{1}, \hat{\boldsymbol{\eta}}_{2}, \hat{\hat{\boldsymbol{\eta}}}_{1}$, and $\hat{\hat{\eta}}_{2}$ with minimum norm such that

$$
\begin{aligned}
& \overline{\mathbf{a}}_{12 m}=\left(\mathbf{R}\left(\hat{\mathbf{n}}, \theta_{1}\right)-\mathbf{I}\right) \hat{\boldsymbol{\eta}}_{1}=\left(\mathbf{R}\left(\hat{\mathbf{n}}, \theta_{1}+\tilde{\theta}_{1}\right)-\mathbf{I}\right) \hat{\hat{\boldsymbol{\eta}}}_{1} \\
& \overline{\mathbf{a}}_{13 m}=\left(\mathbf{R}\left(\hat{\mathbf{n}}, \theta_{2}\right)-\mathbf{I}\right) \hat{\boldsymbol{\eta}}_{2}=\left(\mathbf{R}\left(\hat{\mathbf{n}}, \theta_{2}+\tilde{\theta}_{2}\right)-\mathbf{I}\right) \hat{\hat{\boldsymbol{\eta}}}_{2} .
\end{aligned}
$$

Since $\overline{\mathbf{a}}_{12 m} \neq 0, \overline{\mathbf{a}}_{13 m} \neq 0$, the vectors $\hat{\boldsymbol{\eta}}_{i}, \hat{\hat{\boldsymbol{\eta}}}_{i}, i=1,2$, are all orthogonal to $\hat{\mathbf{n}}$. From the properties of pseudoinverse, we have

$$
(\mathbf{R}(\hat{\mathbf{n}}, \theta)-\mathbf{I})^{+}(\mathbf{R}(\hat{\mathbf{n}}, \theta)-\mathbf{I}) \mathbf{v}=\mathbf{v}
$$

for all vector $\mathbf{v}$ orthogonal to $\hat{\mathbf{n}}$. As a result, substituting the above formulas (27), (28) in (15) and 
(26), the estimated baseline vectors $\hat{\mathbf{a}}_{\perp \mathbf{n}}$ and $\hat{\mathbf{a}}_{\perp \mathbf{n}}$ can be expressed as

$$
\begin{aligned}
& \hat{\mathbf{a}}_{\perp \mathbf{n}}=\alpha \hat{\boldsymbol{\eta}}_{1}+(1-\alpha) \hat{\boldsymbol{\eta}}_{2} \\
& \hat{\mathbf{a}}_{\perp \mathbf{n}}=\alpha \hat{\hat{\boldsymbol{\eta}}}_{1}+(1-\alpha) \hat{\hat{\boldsymbol{\eta}}}_{2} .
\end{aligned}
$$

From (27), we have

$$
\begin{aligned}
\hat{\hat{\boldsymbol{\eta}}}_{1} & =\left(\mathbf{R}\left(\hat{\mathbf{n}}, \theta_{1}+\tilde{\theta}_{1}\right)-\mathbf{I}\right)^{+}\left(\mathbf{R}\left(\hat{\mathbf{n}}, \theta_{1}\right)-\mathbf{I}\right) \hat{\boldsymbol{\eta}}_{1} \\
& =\left(\mathbf{R}\left(\hat{\mathbf{n}}, \theta_{1}+\tilde{\theta}_{1}\right)-\mathbf{I}\right)^{+}\left(\mathbf{R}\left(\hat{\mathbf{n}}, \theta_{1}+\tilde{\theta}_{1}\right) \mathbf{R}\left(\hat{\mathbf{n}},-\tilde{\theta}_{1}\right)-\mathbf{I}\right) \hat{\boldsymbol{\eta}}_{1} .
\end{aligned}
$$

If the error of the rotation angle is infinitesimal, the rotation matrices can be approximated as

$$
\mathbf{R}(\hat{\mathbf{n}}, \tilde{\theta}) \cong \mathbf{I}-\tilde{\theta}[|\hat{\mathbf{n}}|] .
$$

With the above formula, (31) can be approximated as

$$
\begin{aligned}
\hat{\boldsymbol{\eta}}_{1} \cong & \left(\mathbf{R}\left(\hat{\mathbf{n}}, \theta_{1}+\tilde{\theta}_{1}\right)-\mathbf{I}\right)^{+}\left(\mathbf{R}\left(\hat{\mathbf{n}}, \theta_{1}+\tilde{\theta}_{1}\right)\left(\mathbf{I}+\tilde{\theta}_{1}[|\hat{\mathbf{n}}|]\right)-\mathbf{I}\right) \hat{\boldsymbol{\eta}}_{1} \\
= & \left(\mathbf{R}\left(\hat{\mathbf{n}}, \theta_{1}+\tilde{\theta}_{1}\right)-\mathbf{I}\right)^{+}\left(\mathbf{R}\left(\hat{\mathbf{n}}, \theta_{1}+\tilde{\theta}_{1}\right)-\mathbf{I}\right) \hat{\boldsymbol{\eta}}_{1} \\
& +\tilde{\theta}_{1}\left(\mathbf{R}\left(\hat{\mathbf{n}}, \theta_{1}+\tilde{\theta}_{1}\right)-\mathbf{I}\right)^{+} \mathbf{R}\left(\hat{\mathbf{n}}, \theta_{1}+\tilde{\theta}_{1}\right)[|\hat{\mathbf{n}}|] \hat{\boldsymbol{\eta}}_{1} \\
= & \hat{\boldsymbol{\eta}}_{1}+\tilde{\theta}_{1}\left(\mathbf{R}\left(\hat{\mathbf{n}}, \theta_{1}+\tilde{\theta}_{1}\right)-\mathbf{I}\right)^{+} \mathbf{R}\left(\hat{\mathbf{n}}, \theta_{1}+\tilde{\theta}_{1}\right)[|\hat{\mathbf{n}}|] \hat{\boldsymbol{\eta}}_{1} \\
\cong & \hat{\boldsymbol{\eta}}_{1}+\tilde{\theta}_{1}\left(\mathbf{R}\left(\hat{\mathbf{n}}, \theta_{1}\right)-\mathbf{I}\right)^{+} \mathbf{R}\left(\hat{\mathbf{n}}, \theta_{1}\right)[|\hat{\mathbf{n}}|] \hat{\boldsymbol{\eta}}_{1} .
\end{aligned}
$$

Hence, the difference between $\hat{\boldsymbol{\eta}}_{1}$ and $\hat{\boldsymbol{\eta}}_{1}$ is

$$
\tilde{\boldsymbol{\eta}}_{1} \triangleq \hat{\hat{\boldsymbol{\eta}}}_{1}-\hat{\boldsymbol{\eta}}_{1} \cong \tilde{\theta}_{1}\left(\mathbf{R}\left(\hat{\mathbf{n}}, \theta_{1}\right)-\mathbf{I}\right)^{+} \mathbf{R}\left(\hat{\mathbf{n}}, \theta_{1}\right)[|\hat{\mathbf{n}}|] \hat{\boldsymbol{\eta}}_{1} .
$$

With the same process, we have

$$
\tilde{\boldsymbol{\eta}}_{2} \triangleq \hat{\hat{\boldsymbol{\eta}}}_{2}-\hat{\boldsymbol{\eta}}_{2} \cong \tilde{\theta}_{2}\left(\mathbf{R}\left(\hat{\mathbf{n}}, \theta_{2}\right)-\mathbf{I}\right)^{+} \mathbf{R}\left(\hat{\mathbf{n}}, \theta_{2}\right)[|\hat{\mathbf{n}}|] \hat{\boldsymbol{\eta}}_{2} .
$$

Based on (32) and (33), the error due to the imprecise rotations should be

$$
\begin{aligned}
\tilde{\mathbf{a}}_{\perp \mathbf{n}} \triangleq & \hat{\mathbf{a}}_{\perp \mathbf{n}}-\hat{\mathbf{a}}_{\perp \mathbf{n}}=\alpha \tilde{\boldsymbol{\eta}}_{1}+(1-\alpha) \tilde{\boldsymbol{\eta}}_{2} \\
\cong & \alpha \tilde{\theta}_{1}\left(\mathbf{R}\left(\hat{\mathbf{n}}, \theta_{1}\right)-\mathbf{I}\right)^{+} \mathbf{R}\left(\hat{\mathbf{n}}, \theta_{1}\right)[|\hat{\mathbf{n}}|] \hat{\boldsymbol{\eta}}_{1} \\
& +(1-\alpha) \tilde{\theta}_{2}\left(\mathbf{R}\left(\hat{\mathbf{n}}, \theta_{2}\right)-\mathbf{I}\right)^{+} \mathbf{R}\left(\hat{\mathbf{n}}, \theta_{2}\right)[|\hat{\mathbf{n}}|] \hat{\boldsymbol{\eta}}_{2} .
\end{aligned}
$$

The above formula then can be used to estimate the corresponding errors in Algorithm 3 due to rotation errors.

Next, the error analysis is extended to Algorithm 1 and 2. In Algorithm 2, if (18a) is the desired estimate, we have

$$
\begin{aligned}
\hat{\hat{\mathbf{a}}}= & \hat{\mathbf{a}}_{\perp \mathbf{n}}+\sqrt{l^{2}-\left|\hat{\mathbf{a}}_{\perp \mathbf{n}}\right|^{2}} \hat{\mathbf{n}} \\
= & \hat{\mathbf{a}}_{\perp \mathbf{n}}+\tilde{\mathbf{a}}_{\perp \mathbf{n}}+\sqrt{l^{2}-\left|\hat{\mathbf{a}}_{\perp \mathbf{n}}\right|^{2}} \hat{\mathbf{n}} \\
& +\left(\sqrt{l^{2}-\left|\hat{\mathbf{a}}_{\perp \mathbf{n}}+\tilde{\mathbf{a}}_{\perp \mathbf{n}}\right|^{2}}-\sqrt{l^{2}-\left|\hat{\mathbf{a}}_{\perp \mathbf{n}}\right|^{2}}\right) \hat{\mathbf{n}} .
\end{aligned}
$$

The error due to the imprecise rotation about the axis $\mathbf{n}$ is

$$
\tilde{\mathbf{a}} \triangleq \hat{\hat{\mathbf{a}}}-\hat{\mathbf{a}}=\tilde{\mathbf{a}}_{\perp \mathbf{n}}+\left(\sqrt{l^{2}-\left|\hat{\mathbf{a}}_{\perp \mathbf{n}}+\tilde{\mathbf{a}}_{\perp \mathbf{n}}\right|^{2}}-\sqrt{l^{2}-\left|\hat{\mathbf{a}}_{\perp \mathbf{n}}\right|^{2}}\right) \hat{\mathbf{n}} .
$$

On the other hand, if (18b) is chosen, then,

$$
\tilde{\mathbf{a}} \triangleq \hat{\hat{\mathbf{a}}}-\hat{\mathbf{a}}=\tilde{\mathbf{a}}_{\perp \mathbf{n}}-\left(\sqrt{l^{2}-\left|\hat{\mathbf{a}}_{\perp \mathbf{n}}+\tilde{\mathbf{a}}_{\perp \mathbf{n}}\right|^{2}}-\sqrt{l^{2}-\left|\hat{\mathbf{a}}_{\perp \mathbf{n}}\right|^{2}}\right) \hat{\mathbf{n}} .
$$

The above two formulae can be used to evaluate the effect of rotation errors in Algorithm 2.

By a similar process, the error induced by the axis $\mathbf{m}$ rotation errors can be shown to be

$$
\begin{aligned}
\tilde{\mathbf{a}}_{\perp \mathbf{m}}= & \beta \tilde{\varphi}_{1}\left(\mathbf{R}\left(\hat{\mathbf{m}}, \varphi_{1}\right)-\mathbf{I}\right)^{+} \mathbf{R}\left(\hat{\mathbf{m}}, \varphi_{1}\right)[|\hat{\mathbf{m}}|] \hat{\boldsymbol{\zeta}}_{1} \\
& +(1-\beta) \tilde{\varphi}_{2}\left(\mathbf{R}\left(\hat{\mathbf{m}}, \varphi_{2}\right)-\mathbf{I}\right)^{+} \mathbf{R}\left(\hat{\mathbf{m}}, \varphi_{2}\right)[|\hat{\mathbf{m}}|] \hat{\boldsymbol{\zeta}}_{2}
\end{aligned}
$$

where $\hat{\zeta}_{1}, \hat{\zeta}_{2}$ are defined similar to $\hat{\boldsymbol{\eta}}_{1}, \hat{\boldsymbol{\eta}}_{2}$. The effects of rotation errors in Algorithm 1 can be then assessed in the same ways as for Algorithm 2.

EXAMPLE 1 Consider the case of Algorithm 3. Let the rotation angles be $\theta_{1}=60^{\circ}, \theta_{2}=-60^{\circ}$. Assume the estimated rotation axis and the baseline vector are $\hat{\mathbf{n}}=\left[\begin{array}{lll}0 & 0 & 1\end{array}\right]^{\mathrm{T}}$ and $\hat{\mathbf{a}}=\hat{\mathbf{a}}_{\perp \mathbf{n}}=\left[\begin{array}{lll}\cos 45^{\circ} & \sin 45^{\circ} & 0\end{array}\right]^{\mathrm{T}}$, respectively.

a) If the errors of rotation angles are $\tilde{\theta}_{1}=1^{\circ}$, $\tilde{\theta}_{2}=1^{\circ}$, then what are the corresponded errors in $\hat{\mathbf{a}}$ ?

b) If the errors of rotation angles are $\tilde{\theta}_{1}=1^{\circ}$, $\tilde{\theta}_{2}=-1^{\circ}$, then what are the corresponded errors in $\hat{\mathbf{a}}$ ?

Solution:

a) Assume $\alpha=0.5$ and $\hat{\boldsymbol{\eta}}_{1}=\hat{\boldsymbol{\eta}}_{2}=\hat{\mathbf{a}}_{\perp \mathbf{n}}$. Apply (34), the error vector is

$$
\tilde{\mathbf{a}}=\tilde{\mathbf{a}}_{\perp \mathbf{n}} \cong\left[\begin{array}{lll}
0.0062 & -0.0062 & 0
\end{array}\right]^{\mathrm{T}} .
$$

The estimate baseline vector is

$$
\hat{\hat{\mathbf{a}}}=\hat{\mathbf{a}}+\tilde{\mathbf{a}} \cong\left[\begin{array}{lll}
0.7133 & 0.7009 & 0
\end{array}\right]^{\mathrm{T}} .
$$

The error for the azimuth angle is $-0.5^{\circ}$ and the error for the elevation angle is $0^{\circ}$.

b) As before, assume $\alpha=0.5$ and $\hat{\boldsymbol{\eta}}_{1}=\hat{\boldsymbol{\eta}}_{2}=\hat{\mathbf{a}}_{\perp \mathbf{n}}$. Apply (34), the error vector is

$$
\tilde{\mathbf{a}}=\tilde{\mathbf{a}}_{\perp \mathbf{n}} \cong\left[\begin{array}{lll}
-0.0107 & -0.0107 & 0
\end{array}\right]^{\mathrm{T}} .
$$

The estimated baseline vector is

$$
\hat{\hat{\mathbf{a}}}=\hat{\mathbf{a}}+\tilde{\mathbf{a}} \cong\left[\begin{array}{lll}
0.6964 & 0.6964 & 0
\end{array}\right]^{\mathrm{T}} .
$$

The error for the azimuth angle is $0^{\circ}$ and the error for the elevation angle is $0^{\circ}$.

EXAMPLE 2 Consider the case of Algorithm 2.

Let the rotation angles be $\theta_{1}=60^{\circ}, \theta_{2}=-60^{\circ}$.

Assume the estimated rotation axis and the baseline vector are $\hat{\mathbf{n}}=\left[\begin{array}{lll}0 & 0 & 1\end{array}\right]^{\mathrm{T}}$ and $\hat{\mathbf{a}}=$ $\left[\begin{array}{ll}\cos 45^{\circ} \cos 45^{\circ} \sin 45^{\circ} \cos 45^{\circ} \sin 45^{\circ}\end{array}\right]^{\mathrm{T}}$, respectively. 
a) If the errors of rotation angles are $\tilde{\theta}_{1}=1^{\circ}$, $\tilde{\theta}_{2}=1^{\circ}$, then what are the corresponded errors in $\hat{\mathbf{a}}$ ?

b) If the errors of rotation angles are $\tilde{\theta}_{1}=1^{\circ}$, $\tilde{\theta}_{2}=-1^{\circ}$, then what are the corresponded errors in $\hat{\mathbf{a}}$ ?

Solution: Please note that in Algorithm 3, $\hat{\mathbf{n}}$ and $\hat{\mathbf{a}}$ are required to be orthogonal. In Algorithm 2, this restriction is removed.

a) Assume $\alpha=0.5, \hat{\eta}_{1}=\hat{\boldsymbol{\eta}}_{2}=\hat{\mathbf{a}}_{\perp \mathbf{n}}=$ $\left[\cos 45^{\circ} \cos 45^{\circ} \sin 45^{\circ} \cos 45^{\circ} 0\right]$. Apply (34) and (35a)

$$
\begin{aligned}
\tilde{\mathbf{a}}_{\perp \mathbf{n}} & =\left[\begin{array}{lll}
0.0044 & -0.0044 & 0
\end{array}\right]^{\mathrm{T}} \\
\tilde{\mathbf{a}} & \cong\left[\begin{array}{lll}
0.0044 & -0.0044 & 0
\end{array}\right]^{\mathrm{T}} \\
\hat{\hat{\mathbf{a}}} & =\hat{\mathbf{a}}+\tilde{\mathbf{a}} \cong\left[\begin{array}{lll}
0.5044 & 0.4956 & 0.7071
\end{array}\right]^{\mathrm{T}} .
\end{aligned}
$$

Comparing with $\hat{\mathbf{a}}$, the error for the azimuth angle is $-0.5^{\circ}$, and the error for the elevation angle is $-0.0022^{\circ}$.

b) Again, assume $\alpha=0.5, \hat{\boldsymbol{\eta}}_{1}=\hat{\boldsymbol{\eta}}_{2}=\hat{\mathbf{a}}_{\perp \mathbf{n}}=$ $\left[\cos 45^{\circ} \cos 45^{\circ} \sin 45^{\circ} \cos 45^{\circ} 0\right]$. Apply (34) and (35a)

$$
\begin{aligned}
\tilde{\mathbf{a}}_{\perp \mathbf{n}} & =\left[\begin{array}{lll}
-0.0076 & -0.0076 & 0
\end{array}\right]^{\mathrm{T}} \\
\tilde{\mathbf{a}} & \cong\left[\begin{array}{lll}
-0.0076 & -0.0076 & 0.0105
\end{array}\right]^{\mathrm{T}} \\
\hat{\hat{\mathbf{a}}} & =\hat{\mathbf{a}}+\tilde{\mathbf{a}} \cong\left[\begin{array}{lll}
0.4924 & 0.4924 & 0.7176
\end{array}\right]^{\mathrm{T}} .
\end{aligned}
$$

Comparing with $\hat{\mathbf{a}}$, the error for the azimuth angle is $0^{\circ}$, and the error for the elevation angle is $0.8596^{\circ}$.

\section{CONCLUSIONS}

Based on the concept of space difference, the baseline rotation method using GPS carrier phase measurements for direction finding has been proposed. Three algorithms associated with different configurations have been investigated and can be used to resolve the double-difference ambiguities. Algorithms 1 and 2 deal with the direction of a baseline vector $\mathbf{a}$, which can be rotated about rotation axes $\mathbf{n}$ and $\mathbf{m}$. In Algorithm 2, the relationships among $\mathbf{a}, \mathbf{m}$, and $\mathbf{n}$ are unknown. Two rotations about the axis $\mathbf{n}$ and two rotations about the axis $\mathbf{m}$ are required in order to find the directions of $\mathbf{a}, \mathbf{n}$, and $\mathbf{m}$, respectively. The application of Algorithm 1 can be used to obtain the LOS of a tracking radar. In Algorithm 2, it is assumed that the rotation axes $\mathbf{n}$ and $\mathbf{m}$ are orthogonal, and the rotation axis $\mathbf{m}$ and the baseline vector a are orthogonal as well. Here, two rotations about the axis $\mathbf{n}$ and one rotation about the axis $\mathbf{m}$ are needed to find the directions. Algorithm 2 can be applied to obtain the aiming directions of artillery rockets. Algorithm 3 deals with the direction of a baseline vector $\mathbf{a}$, which can be rotated about a rotation axis $\mathbf{n}$. In this algorithm, a and $\mathbf{n}$ are assumed to be orthogonal. Two rotations about the axis $\mathbf{n}$ are then necessary in order to find $\mathbf{a}$ and $\mathbf{n}$. Software simulations and hardware experiments of Algorithm 2 have been conducted. For a $1.35 \mathrm{~m}$ baseline, results of using Motorola ONCORE receivers show that the sample standard deviations of the azimuth and elevation angles of the determined baseline are $0.91^{\circ}$ and $1.23^{\circ}$, respectively. The errors caused by the rotating mechanisms are unavoidable. The propagation of the rotation errors will affect the accuracy of the direction finding. The concept of orthogonality between the null space of the adjoint linear transformation and the range space of the linear transformation has been used in the error analysis. Furthermore, two numerical examples are used to illustrate our analysis. The assumption of no cycle slip occurring during the time intervals of baseline rotations must be satisfied in our algorithms. Otherwise, cycle slip detection and compensation methods may be used to ensure that the above assumption is met.

\section{REFERENCES}

[1] Hofmann-Wellenhof, B., Lichtenegger, H., and Collins, J. (1992)

Global Positioning System: Theory and Practice. New York: Springer-Verlag, 1992.

[2] Cohen, C. E. (1992) Attitude determination using GPS: Development of an all solid-state guidance, navigation, and control sensor for air and space vehicles based on the global positioning system.

Ph.D. dissertation, Department of Aeronautics and Astronautics, Stanford University, Stanford, CA, Dec. 1992.

[3] Brown, R. A. (1992)

Instantaneous GPS attitude determination

IEEE Aerospace and Electronics Magazine, 7 (June 1992), 3-8.

[4] Yang, C. C. (1994)

GPS phase double difference method for single axis attitude determination.

M.S. thesis, Department of Electrical Engineering, National Taiwan University, Taipei, Taiwan, R.O.C., June 1994.

[5] Juang, J. C., and Huang, G. S. (1997)

Development of GPS-based attitude determination algorithms.

IEEE Transactions on Aerospace and Electronic Systems, 33 (July 1997), 968-976.

[6] Brown, R., and Ward, P. (1990)

A GPS receiver with built-in precision pointing capability. In Proceedings of IEEE Position Location and Navigation Symposium, Las Vegas, NV, Mar. 1990, 83-93.

[7] Remondi, B. W. (1986) Performing centimeter-level surveys in seconds with GPS carrier phase: Initial results.

In Proceedings of the Fourth International Geodetic Symposium on Satellite Positioning, Vol. 2, Austin, TX, Apr. 1986, 1229-1249.

[8] Tu, C. H., Wang, L. S., Tu, K. Y., and Chang, F. R. (1997) GPS compass: A novel navigation equipment. IEEE Transactions on Aerospace and Electronic Systems, 33 (July 1997), 1063-1068. 

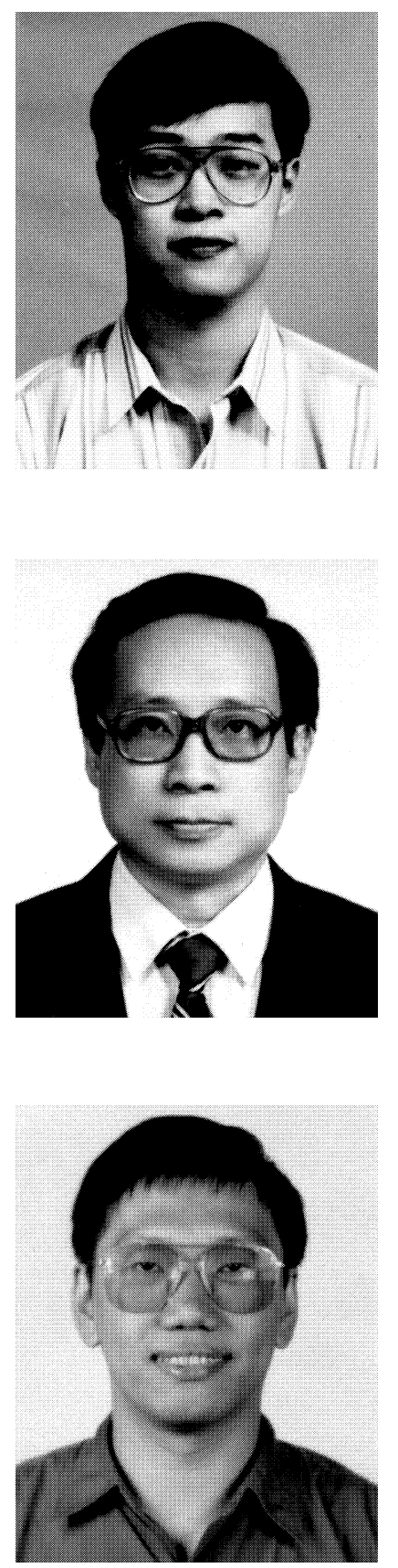

Hsin-Min Peng was born in Taiwan, Republic of China in 1971. He received the B.S. and the Ph.D. degrees from National Taiwan University, Taipei, Taiwan, in 1994 and 1999, respectively, both in electrical engineering.

He is currently in military service. His research interests include navigation and attitude determination via Global Positioning System.

Fan-Ren Chang was born in Taiwan, Republic of China in 1949. He received the B.S. and M.S. degrees from National Chiao Tung University, Taiwan, in 1972 and 1974, respectively, the Ph.D. degree from University of Houston, Houston, TX, in 1985, all in electrical engineering.

From 1976 to 1981, he was an assistant researcher of Chung Shan Institute of Science and Technology. He worked for missile and fire control system projects. He joined the Department of Electrical Engineering, National Taiwan University in 1985 as an Associate Professor. Since 1990, he has been a Professor at the same department. His current research interests include linear multivariable system, generalized system, and applications of Global Positioning System.

Li-Sheng Wang was born in Taiwan, Republic of China in 1961. He received the B.S. degree from National Taiwan University, Taipei, Taiwan, in 1983, and the M.S. and Ph.D. degrees from the University of Maryland, College Park, in 1987 and 1990, respectively, all in electrical engineering.

From 1990 to 1991, he held a research and teaching position at the Department of Mathematics, University of Maryland, College Park. Since 1991, he has been with the Institute of Applied Mechanics, National Taiwan University, where he is currently a Professor.

Dr. Wang currently serves as an Associate Editor for IEEE Transactions on Automatic Control. His research interests include nonlinear systems theory, guidance and control, geometrical mechanics, robotics, and aerospace applications. 